\title{
Temporal variation for fractional heat equations with additive white noise
}

\author{
Jing Cui ${ }^{1}$, Yumiao Li ${ }^{2}$ and Litan Yan²
}

${ }^{\text {*Correspondence: }}$

litan-yan@hotmail.com

${ }^{2}$ Department of Mathematics, Donghua University, 2999 North Renmin Rd., Songjiang, Shanghai, 201620, P.R. China

Full list of author information is available at the end of the article

\section{Abstract}

Let $u(t, x)$ be the solution to a stochastic heat equation

$$
\frac{\partial}{\partial t} u=\Delta_{\alpha} u+\frac{\partial^{2}}{\partial t \partial x} B(t, x), \quad t \geq 0, x \in \mathbb{R}
$$

with initial condition $u(0, x) \equiv 0$, where $B$ is a time-space white noise, $\Delta_{\alpha}=-(-\Delta)^{\alpha / 2}$ is the fractional Laplacian with $\alpha \in(1,2]$. In this paper we study the quadratic variation of the process $W^{\alpha}=\left\{W_{t}^{\alpha}=u(t, \cdot), t \geq 0\right\}$. We construct a Banach space $\mathscr{H}$ of measurable functions such that the generalized quadratic covariation $\left\langle f\left(W^{\alpha}\right), W^{\alpha}\right\rangle^{(\alpha)}$ of $f\left(W^{\alpha}\right)$ and $W^{\alpha}$ defined by

$$
\left\langle f\left(W^{\alpha}\right),\left.W^{\alpha}\right|_{t} ^{(\alpha)}:=\lim _{\varepsilon \downarrow 0} \frac{1}{\varepsilon^{\frac{\alpha-1}{2 \alpha}}} \int_{0}^{t}\left\{f\left(W_{s+\varepsilon, x}^{\alpha}\right)-f\left(W_{s}^{\alpha}\right)\right\}\left(W_{s+\varepsilon, x}^{\alpha}-W_{s}^{\alpha}\right) d s^{\frac{\alpha-1}{2 \alpha}}\right.
$$

exists in $L^{2}(\Omega)$, provided $f \in \mathscr{H}$. Moreover, we consider some related questions.

MSC: $60 \mathrm{G} 15 ; 60 \mathrm{H} 05 ; 60 \mathrm{H} 15$

Keywords: fractional heat equation; stochastic integral; Itô formula; quadratic covariation; local time

\section{Introduction and main results}

Recently, Swanson [1] (see also Pospisil and Tribe [2]) studied the exact variations of the solution to the following one-dimensional stochastic heat equation:

$$
\frac{\partial}{\partial t} u=\frac{1}{2} \Delta u+\frac{\partial^{2}}{\partial t \partial x} B(t, x), \quad t \geq 0, x \in \mathbb{R}
$$

with initial condition $u(0, x)=0$, where $B$ is a time-space white noise on $[0, \infty) \times \mathbb{R}$. It is clear that the solution can be characterized as

$$
u(t, x)=\int_{0}^{t} \int_{\mathbb{R}} p(t-s, x-y) B(d s, d y)
$$

here $p(t, x)=\frac{1}{\sqrt{4 \pi t}} e^{-\frac{x^{2}}{4 t}}$ is the heat kernel of Lapalacian. Under the circumstance, we know (see Swanson [1]) that

$$
E[u(t, x) u(s, x)]=\frac{1}{\sqrt{2 \pi}}\left((t+s)^{1 / 2}-|t-s|^{1 / 2}\right), \quad t, s \geq 0,
$$

(c) 2016 Cui et al. This article is distributed under the terms of the Creative Commons Attribution 4.0 International License (http://creativecommons.org/licenses/by/4.0/), which permits unrestricted use, distribution, and reproduction in any medium, provided you give appropriate credit to the original author(s) and the source, provide a link to the Creative Commons license, and indicate if changes were made. 
moreover, the process $t \mapsto u(t, x)$ has a nontrivial quartic variation. As a general conclusion from these results, in Sun et al. [3] we considered the quadratic covariations and Itô's formula for the solution. The above results show that the process $u=\{u(t, x)\}$ as a noise admits the next special structures. For more results for an Itô analysis of stochastic heat equations one refers to Da Prato et al. [4], Deya and Tindel [5], Denis [6], Gradinaru et al. [7], Ouahhabi and Tudor [8], León and Tindel [9], Torres et al. [10], Tudor and Xiao [11], Ciprian and Tudor [12], Wu [13], Zambotti [14], and the references therein.

In the present paper, we study the temporal variation of the solution $\{u(t, x), t \geq 0, x \in \mathbb{R}\}$ to the following fractional stochastic heat equation:

$$
\frac{\partial}{\partial t} u=\Delta_{\alpha} u+\frac{\partial^{2}}{\partial t \partial x} B(t, x), \quad t \geq 0, x \in \mathbb{R}
$$

with initial condition $u(0, x)=0$, where $X$ is a time-space white noise on $[0, \infty) \times \mathbb{R}, \Delta_{\alpha}=$ $-(-\Delta)^{\alpha / 2}$ is the fractional Lapalacian with $\alpha \in(1,2]$. Let $p_{\alpha}(t, x, y)$ be the heat kernel of operator $\Delta_{\alpha}$, i.e. the transition density function of one-dimensional symmetry $\alpha$-stable Lévy process. Then the solution $u=\{u(t, x), t \geq 0, x \in \mathbb{R}\}$ is a two parameters Gaussian process and

$$
u(t, x)=\int_{0}^{t} \int_{\mathbb{R}} p_{\alpha}(t-s, x-y) B(d s, d y) .
$$

This paper is organized as follows. In Section 2, we establish some technical estimates associated with the solution based on the heat kernel estimates of the operator $\Delta_{\alpha}=$ $-(-\Delta)^{\alpha / 2}$. In Section 3, as some applications of Section 2 we introduce Wiener integrals with respect to the process $W^{\alpha}=\left\{W_{t}^{\alpha}=u(t, \cdot), t \geq 0\right\}$. In Section 4 we show that the process $W^{\alpha}$ admits a nontrivial strong $p=\frac{2 \alpha}{\alpha-1}$-variation, i.e.

$$
\lim _{\varepsilon \downarrow 0} \frac{1}{\varepsilon} \int_{0}^{t}\left|W_{\varepsilon+s}^{\alpha}-W_{s}^{\alpha}\right|^{\frac{2 \alpha}{\alpha-1}} d s=C_{\alpha} t
$$

in probability for every $t>0$. As a related question, we introduce a so-called the generalized quadratic covariation of $f\left(W^{\alpha}\right)$ and $W^{\alpha}$ defined by

$$
\left\langle f\left(W^{\alpha}\right),\left.W^{\alpha}\right|_{t} ^{(\alpha)}:=\lim _{\varepsilon \downarrow 0} \frac{1}{\varepsilon^{\frac{\alpha-1}{2 \alpha}}} \int_{0}^{t}\left\{f\left(W_{s+\varepsilon}^{\alpha}\right)-f\left(W_{s}^{\alpha}\right)\right\}\left(W_{s+\varepsilon}^{\alpha}-W_{s}^{\alpha}\right) d s^{\frac{\alpha-1}{2 \alpha}}\right.
$$

in probability. We construct a Banach space $\mathscr{H}$ of measurable functions such that the generalized quadratic covariation $\left\langle f\left(W^{\alpha}\right), W^{\alpha}\right\rangle^{(\alpha)}$ exists in $L^{2}(\Omega)$, provided $f \in \mathscr{H}$. In Section 5 , we introduce that the Itô's formula and Bouleau-Yor type identity for the temporal process $W^{\alpha}$.

\section{Preliminaries}

In this section, we present some technical estimates associated with the solution

$$
u(t, x)=\int_{0}^{t} \int_{\mathbb{R}} p_{\alpha}(t-r, x-y) B(d r, d y), \quad t \geq 0, x \in \mathbb{R},
$$

with $1<\alpha \leq 2$, where $p_{\alpha}(t, x)$ is the heat kernel of the operator $\Delta_{\alpha}=-(-\Delta)^{\alpha / 2}$. For simplicity, throughout this paper we denote by $C$ a positive constant depending only on the 
subscripts and its value may be different in different places, and this assumption is also suitable for $c$. If there exist positive constants $b_{1}$ and $b_{2}$ such that

$$
b_{1} G(x) \leq F(x) \leq b_{2} G(x)
$$

in the common domain of definition for $F$ and $G$, then we employ the notation $F \asymp G$.

It is clear that the heat kernel $p_{\alpha}(t, x)$ is the fundamental solution of the following equation:

$$
\frac{\partial u}{\partial t}=\Delta_{\alpha} u
$$

$p_{\alpha}(t, x)$ is also the transition density function of one-dimensional symmetry $\alpha$-stable Lévy process $X$. Moreover, $p_{\alpha}(t, x)$ satisfies (see, for example, Blumenthal and Getoor [15])

$$
\int_{\mathbb{R}} p_{\alpha}(t, x) e^{i x \xi} d x=e^{-t|\xi|^{\alpha}}, \quad \xi \in \mathbb{R}, t \geq 0
$$

When $\alpha=1$, we get

$$
p_{1}(t, x)=\frac{t}{\pi\left(x^{2}+t^{2}\right)} .
$$

For all $x \in \mathbb{R}$ and $t>0$, we have

$$
p_{\alpha}(t, x)=t^{-\frac{1}{\alpha}} p_{\alpha}\left(1, x t^{-\frac{1}{\alpha}}\right) .
$$

Moreover, for all $x \in \mathbb{R}$ and $t>0$, we obtain

$$
c^{-1}\left(\frac{t}{|x|^{1+\alpha}} \wedge t^{-\frac{1}{\alpha}}\right) \leq p_{\alpha}(t, x) \leq c\left(\frac{t}{|x|^{1+\alpha}} \wedge t^{-\frac{1}{\alpha}}\right),
$$

where $c>1$ is a constant.

Now, we present some estimates. Denote by $W^{\alpha}=\left\{W_{t}^{\alpha}=u(t, \cdot), t \geq 0\right\}$ the temporal process. For $x \in \mathbb{R}$ and all $t, s>0$, we have

$$
\begin{aligned}
E\left(W_{t}^{\alpha} W_{s}^{\alpha}\right) & =\int_{0}^{s \wedge t} \int_{\mathbb{R}} p_{\alpha}(t-r, x-z) p_{\alpha}(s-r, x-z) d z d r \\
& =\int_{0}^{s \wedge t} p_{\alpha}(t+s-2 r, 0) d r \\
& =\frac{\Gamma(1 / \alpha)}{\alpha \pi} \int_{0}^{s \wedge t}(t+s-2 r)^{-\frac{1}{\alpha}} d r \\
& =\frac{\Gamma(1 / \alpha)}{2 \pi(\alpha-1)}\left((t+s)^{\frac{\alpha-1}{\alpha}}-|t-s|^{\frac{\alpha-1}{\alpha}}\right) .
\end{aligned}
$$

Lemma 2.1 For $x \in \mathbb{R}$ and all $t, s>0$, we have

$$
E\left[\left(W_{t}^{\alpha}-W_{s}^{\alpha}\right)^{2}\right] \asymp(t-s)^{\frac{\alpha-1}{\alpha}} .
$$


Proof For $x \in[0,1]$, let

$$
f(x)=1-2^{\frac{1}{\alpha}}\left((1+x)^{\frac{\alpha-1}{\alpha}}-(1-x)^{\frac{\alpha-1}{\alpha}}\right)+x^{\frac{\alpha-1}{\alpha}} .
$$

Then, for $x \in[0,1]$, we have

$$
f(x) \asymp(1-x)^{\frac{\alpha-1}{\alpha}} .
$$

It follows that

$$
\begin{aligned}
E\left[\left(W_{t}^{\alpha}-W_{s}^{\alpha}\right)^{2}\right] & =\frac{\Gamma(1 / \alpha)}{2^{\frac{1}{\alpha}} \pi(\alpha-1)}\left(t^{\frac{\alpha-1}{\alpha}}-2^{\frac{1}{\alpha}}\left((t+s)^{\frac{\alpha-1}{\alpha}}-(t-s)^{\frac{\alpha-1}{\alpha}}\right)+s^{\frac{\alpha-1}{\alpha}}\right) \\
& =\frac{\Gamma(1 / \alpha)}{2^{\frac{1}{\alpha}} \pi(\alpha-1)} t^{\frac{\alpha-1}{\alpha}} f(s / t) \asymp(t-s)^{\frac{\alpha-1}{\alpha}}
\end{aligned}
$$

for all $t>s>0$.

Lemma 2.2 For $x \in \mathbb{R}$ and all $t, s>0$, we have

$$
\left|E\left[W_{r}^{\alpha}\left(W_{t}^{\alpha}-W_{s}^{\alpha}\right)\right]\right| \leq \frac{\Gamma(1 / \alpha)}{\pi(\alpha-1)}|t-s|^{\frac{\alpha-1}{\alpha}} .
$$

Proof By an elementary calculation, we have

$$
\begin{aligned}
\left|E\left[W_{r}^{\alpha}\left(W_{t}^{\alpha}-W_{s}^{\alpha}\right)\right]\right| & =\left|E\left(W_{r}^{\alpha} W_{t}^{\alpha}\right)-E\left(W_{r}^{\alpha} W_{s}^{\alpha}\right)\right| \\
& =\frac{\Gamma(1 / \alpha)}{2 \pi(\alpha-1)}\left|(t+r)^{\frac{\alpha-1}{\alpha}}-\right| t-\left.r\right|^{\frac{\alpha-1}{\alpha}}-(s+r)^{\frac{\alpha-1}{\alpha}}+|s-r|^{\frac{\alpha-1}{\alpha}} \mid \\
& \leq \frac{\Gamma(1 / \alpha)}{2 \pi(\alpha-1)}\left|(t+r)^{\frac{\alpha-1}{\alpha}}-(s+r)^{\frac{\alpha-1}{\alpha}}\right|+|| t-\left.r\right|^{\frac{\alpha-1}{\alpha}}-|s-r|^{\frac{\alpha-1}{\alpha}} \mid \\
& \leq \frac{\Gamma(1 / \alpha)}{\pi(\alpha-1)}|t-s|^{\frac{\alpha-1}{\alpha}}
\end{aligned}
$$

for $x \in \mathbb{R}$ and all $t, s, r>0$.

Lemma 2.3 For all $t>s>t^{\prime}>s^{\prime}>0$ and $x \in \mathbb{R}$ we have

$$
\left|E\left[\left(W_{t}^{\alpha}-W_{s}^{\alpha}\right)\left(W_{t^{\prime}}^{\alpha}-W_{s^{\prime}}^{\alpha}\right)\right]\right| \leq C \frac{\left[(t-s)\left(t^{\prime}-s^{\prime}\right)\right]^{\frac{\alpha-1}{\alpha}}}{\left(s-t^{\prime}\right)^{\frac{\alpha-1}{\alpha}}} .
$$

Proof By applying the mean value theorem, there exist some $\xi \in(s, t)$ and $\eta \in\left(s^{\prime}, t^{\prime}\right)$ such that

$$
\begin{aligned}
g_{1} & :=\left(\left(t+t^{\prime}\right)^{\frac{\alpha-1}{\alpha}}-\left(t+s^{\prime}\right)^{\frac{\alpha-1}{\alpha}}\right)-\left(\left(s+t^{\prime}\right)^{\frac{\alpha-1}{\alpha}}-\left(s+s^{\prime}\right)^{\frac{\alpha-1}{\alpha}}\right) \\
& =\frac{\alpha-1}{\alpha}(t-s)\left(\left(\xi+t^{\prime}\right)^{-\frac{1}{\alpha}}-\left(\xi+s^{\prime}\right)^{-\frac{1}{\alpha}}\right) \\
& =-\frac{\alpha-1}{\alpha^{2}}(t-s)\left(t^{\prime}-s^{\prime}\right)(\xi+\eta)^{-1-\frac{1}{\alpha}},
\end{aligned}
$$


which gives

$$
\left|g_{1}\right| \leq \frac{\alpha-1}{\alpha^{2}}(t-s)\left(t^{\prime}-s^{\prime}\right)\left(s+s^{\prime}\right)^{-1-\frac{1}{\alpha}}
$$

By a similar argument, we get

$$
\begin{aligned}
\left|g_{2}\right| & :=\left|\left(\left(t-s^{\prime}\right)^{\frac{\alpha-1}{\alpha}}-\left(t-t^{\prime}\right)^{\frac{\alpha-1}{\alpha}}\right)-\left(\left(s-s^{\prime}\right)^{\frac{\alpha-1}{\alpha}}-\left(s-t^{\prime}\right)^{\frac{\alpha-1}{\alpha}}\right)\right| \\
& =\frac{\alpha-1}{\alpha}(t-s)\left|\left(\xi-s^{\prime}\right)^{-\frac{1}{\alpha}}-\left(\xi-t^{\prime}\right)^{-\frac{1}{\alpha}}\right| \\
& =\frac{\alpha-1}{\alpha^{2}}(t-s)\left(t^{\prime}-s^{\prime}\right)(\xi-\eta)^{-1-\frac{1}{\alpha}} \leq \frac{\alpha-1}{\alpha^{2}}(t-s)\left(t^{\prime}-s^{\prime}\right)\left(s-t^{\prime}\right)^{-1-\frac{1}{\alpha}}
\end{aligned}
$$

for some $\eta \in\left(s^{\prime}, t^{\prime}\right)$ and $\xi \in(s, t)$. It follows that

$$
\begin{aligned}
\left|E\left[\left(W_{t}^{\alpha}-W_{s}^{\alpha}\right)\left(W_{t^{\prime}}^{\alpha}-W_{s^{\prime}}^{\alpha}\right)\right]\right| & =\frac{\Gamma(1 / \alpha)}{2 \pi(\alpha-1)}\left|g_{1}+g_{2}\right| \\
& \leq \frac{\Gamma(1 / \alpha)}{\pi \alpha^{2}}(t-s)\left(t^{\prime}-s^{\prime}\right)\left(s-t^{\prime}\right)^{-1-\frac{1}{\alpha}}
\end{aligned}
$$

for all $t>s>t^{\prime}>s^{\prime}>0$. On the other hand, noting that

$$
\left|E\left[\left(W_{t}^{\alpha}-W_{s}^{\alpha}\right)\left(W_{t^{\prime}}^{\alpha}-W_{s^{\prime}}^{\alpha}\right)\right]\right| \leq \sqrt{\frac{\Gamma(1 / \alpha)}{\pi(\alpha-1)}}\left[(t-s)\left(t^{\prime}-s^{\prime}\right)\right]^{\frac{\alpha-1}{2 \alpha}},
$$

it follows that

$$
\frac{\left|E\left[\left(W_{t}^{\alpha}-W_{s}^{\alpha}\right)\left(W_{t^{\prime}}^{\alpha}-W_{s^{\prime}}^{\alpha}\right)\right]\right|}{\sqrt{\frac{\Gamma(1 / \alpha)}{\pi(\alpha-1)}}\left[(t-s)\left(t^{\prime}-s^{\prime}\right)\right]^{\frac{\alpha-1}{2 \alpha}}} \leq\left(\frac{\left|E\left[\left(W_{t}^{\alpha}-W_{s}^{\alpha}\right)\left(W_{t^{\prime}}^{\alpha}-W_{s^{\prime}}^{\alpha}\right)\right]\right|}{\sqrt{\frac{\Gamma(1 / \alpha)}{\pi(\alpha-1)}}\left[(t-s)\left(t^{\prime}-s^{\prime}\right)\right]^{\frac{\alpha-1}{2 \alpha}}}\right)^{\gamma}
$$

for all $\beta \in[0,1]$. By this, together with (2.6), we obtain

$$
\begin{aligned}
\mid E & {\left[\left(W_{t}^{\alpha}-W_{s}^{\alpha}\right)\left(W_{t^{\prime}}^{\alpha}-W_{s^{\prime}}^{\alpha}\right)\right] \mid } \\
& \leq C\left[(t-s)\left(t^{\prime}-s^{\prime}\right)\right]^{\frac{\alpha-1}{2 \alpha}(1-\gamma)}\left|E\left[\left(W_{t}^{\alpha}-W_{s}^{\alpha}\right)\left(W_{t^{\prime}}^{\alpha}-W_{s^{\prime}}^{\alpha}\right)\right]\right|^{\gamma} \\
& \leq C\left[(t-s)\left(t^{\prime}-s^{\prime}\right)\right]^{\frac{\alpha-1}{2 \alpha}(1-\gamma)+\gamma} \frac{1}{\left(s-t^{\prime}\right)^{\frac{1+\alpha}{\alpha} \gamma}} \\
& =C \frac{\left[(t-s)\left(t^{\prime}-s^{\prime}\right)\right]^{\frac{\alpha-1}{\alpha}}}{\left(s-t^{\prime}\right)^{\frac{\alpha-1}{\alpha}}}
\end{aligned}
$$

and by taking $\gamma=\frac{\alpha-1}{\alpha+1}$, the lemma follows.

Lemma 2.4 For all $t>s>0$, let $\sigma_{t}^{2}=E\left[\left(W_{t}^{\alpha}\right)^{2}\right], \sigma_{s}^{2}=E\left[\left(W_{s}^{\alpha}\right)^{2}\right], \mu_{t, s}=E\left[W_{t}^{\alpha} W_{s}^{\alpha}\right]$. Then we have

$$
\sigma_{t}^{2} \sigma_{s}^{2}-\mu_{t, s}^{2} \asymp[s(t-s)]^{\frac{\alpha-1}{\alpha}}
$$


Proof Let $\kappa_{\alpha}=\frac{\Gamma(1 / \alpha)}{2^{\frac{1}{\alpha}} \pi(\alpha-1)}$ and

$$
G(x)=2^{\frac{2(\alpha-1)}{\alpha}} x^{\frac{\alpha-1}{\alpha}}-\left((1+x)^{\frac{\alpha-1}{\alpha}}-(1-x)^{\frac{\alpha-1}{\alpha}}\right)^{2}
$$

with $x \in[0,1]$. Then

$$
\begin{aligned}
\sigma_{t}^{2} \sigma_{s}^{2}-\mu_{t, s}^{2} & =\kappa_{\alpha}^{2}\left\{(s t)^{\frac{\alpha-1}{\alpha}}-2^{\frac{2(1-\alpha)}{\alpha}}\left((t+s)^{\frac{\alpha-1}{\alpha}}-(t-s)^{\frac{\alpha-1}{\alpha}}\right)^{2}\right\} \\
& =\kappa_{\alpha}^{2} 2^{\frac{2(1-\alpha)}{\alpha}} t^{\frac{2(\alpha-1)}{\alpha}} G(x)
\end{aligned}
$$

for all $t>s>0$ and $x=\frac{s}{t}$. We get

$$
\begin{aligned}
G(x) & =2^{\frac{2(\alpha-1)}{\alpha}} x^{\frac{\alpha-1}{\alpha}}-\left((1+x)^{\frac{\alpha-1}{\alpha}}-(1-x)^{\frac{\alpha-1}{\alpha}}\right)^{2} \\
& =\left\{2^{\frac{\alpha-1}{\alpha}} x^{\frac{\alpha-1}{2 \alpha}}-(1+x)^{\frac{\alpha-1}{\alpha}}+(1-x)^{\frac{\alpha-1}{\alpha}}\right\}\left\{2^{\frac{\alpha-1}{\alpha}} x^{\frac{\alpha-1}{2 \alpha}}+(1+x)^{\frac{\alpha-1}{\alpha}}-(1-x)^{\frac{\alpha-1}{\alpha}}\right\} \\
& \equiv G_{1}(x) G_{2}(x) .
\end{aligned}
$$

It is obvious that

$$
2^{\frac{\alpha-1}{\alpha}} x^{\frac{\alpha-1}{2 \alpha}} \leq G_{2}(x) \leq 2^{1+\frac{\alpha-1}{\alpha}} x^{\frac{\alpha-1}{2 \alpha}} .
$$

We first estimate $G_{1}(x)$. By an elementary calculation we can show that

$$
(u+v-1)^{\frac{\alpha-1}{\alpha}} \leq u^{\frac{\alpha-1}{\alpha}}+v^{\frac{\alpha-1}{\alpha}}-1 \leq(u+v-1)^{\frac{\alpha-1}{\alpha}}+(u v)^{\frac{\alpha-1}{\alpha}}
$$

holds for $u+v \geq 1,0 \leq u, v \leq 1,0 \leq K \leq 1$. By taking

$$
u=\frac{2 \sqrt{x}}{1+x}, \quad v=\frac{1-x}{1+x}
$$

in (2.9), we have

$$
0 \leq u, v \leq 1, \quad u+v-1=\frac{2 \sqrt{x}+(1-x)-(1+x)}{1+x} \geq 0
$$

and

$$
\begin{aligned}
G_{1}(x) & =(1+x)^{\frac{\alpha-1}{\alpha}}\left(\left(\frac{2 \sqrt{x}}{1+x}\right)^{\frac{\alpha-1}{\alpha}}+\left(\frac{1-x}{1+x}\right)^{\frac{\alpha-1}{\alpha}}-1\right) \\
& \leq(1+x)^{\frac{\alpha-1}{\alpha}}\left(\frac{2 \sqrt{x}}{1+x}+\frac{1-x}{1+x}-1\right)^{\frac{\alpha-1}{\alpha}}+(1+x)^{\frac{\alpha-1}{\alpha}}\left(\frac{2 \sqrt{x}}{1+x} \cdot \frac{1-x}{1+x}\right)^{\frac{\alpha-1}{\alpha}} \\
& \leq(2 \sqrt{x}-2 x)^{\frac{\alpha-1}{\alpha}}+(2 \sqrt{x} \cdot(1-x))^{\frac{\alpha-1}{\alpha}} \\
& \leq\left(2 \sqrt{x}-2 x^{3 / 2}\right)^{\frac{\alpha-1}{\alpha}}+(2 \sqrt{x} \cdot(1-x))^{\frac{\alpha-1}{\alpha}} \\
& \leq C x^{\frac{\alpha-1}{2 \alpha}}(1-x)^{\frac{\alpha-1}{\alpha}}
\end{aligned}
$$


for all $x \in[0,1]$. On the other hand, for all $x \in[0,1]$, we have

$$
\begin{aligned}
G_{1}(x) & =(1+x)^{\frac{\alpha-1}{\alpha}}\left(\left(\frac{2 \sqrt{x}}{1+x}\right)^{\frac{\alpha-1}{\alpha}}+\left(\frac{1-x}{1+x}\right)^{\frac{\alpha-1}{\alpha}}-1\right) \\
& \geq(1+x)^{\frac{\alpha-1}{\alpha}}\left(\frac{2 \sqrt{x}}{1+x}+\frac{1-x}{1+x}-1\right)^{\frac{\alpha-1}{\alpha}} \\
& =(2 \sqrt{x}+(1-x)-(1+x))^{\frac{\alpha-1}{\alpha}} \\
& =((1-x)-(1-2 \sqrt{x}+x))^{\frac{\alpha-1}{\alpha}} \\
& =(\sqrt{1-x}-(1-\sqrt{x}))^{\frac{\alpha-1}{\alpha}}(\sqrt{1-x}+(1-\sqrt{x}))^{\frac{\alpha-1}{\alpha}} \\
& \geq(\sqrt{1-x}-(1-\sqrt{x}))^{\frac{\alpha-1}{\alpha}}(1-x)^{\frac{\alpha-1}{2 \alpha}} .
\end{aligned}
$$

Noting that

$$
1=\sqrt{1-x+x} \leq \sqrt{1-x} \vee \sqrt{x}+(\sqrt{2}-1)(\sqrt{1-x} \wedge \sqrt{x})
$$

by using the Bernoulli inequality

$$
(1+x)^{\beta} \leq 1+\beta x^{\beta} \quad(0 \leq x, \beta \leq 1) .
$$

We further get

$$
\begin{aligned}
\sqrt{1-x}+\sqrt{x}-1 & \geq \frac{1}{2} \sqrt{1-x} \wedge \sqrt{x} \\
& \geq \frac{1}{2} \sqrt{(1-x) x}
\end{aligned}
$$

for $0 \leq x \leq 1$. It follows that

$$
G_{1}(x) \geq(\sqrt{1-x}-(1-\sqrt{x}))^{\frac{\alpha-1}{\alpha}}(1-x)^{\frac{\alpha-1}{2 \alpha}} \geq \frac{1}{2^{\frac{\alpha-1}{\alpha}}} x^{\frac{\alpha-1}{2 \alpha}}(1-x)^{\frac{\alpha-1}{\alpha}}
$$

for all $x \in[0,1]$. Therefore, the desired estimates

$$
G(x) \asymp x^{\frac{\alpha-1}{\alpha}}(1-x)^{\frac{\alpha-1}{\alpha}}
$$

hold and the lemma follows.

At the end of this section, we investigate Skorohod integrals associated with the temporal process $W^{\alpha}=\left\{W_{t}^{\alpha}=u(t, \cdot), 0 \leq t \leq T\right\}$. From the previous discussion, we have shown that the process $W^{\alpha}$ is neither a Markov process nor a semimartingale, thus many powerful techniques of stochastic analysis are not available. Noting they are Gaussian processes, so we can develop the stochastic calculus of variations with respect to them. One refers to Alós et al. [16] and Nualart [17] for more details of stochastic calculus for Gaussian processes. 
Denote by $\mathcal{E}$ the set of linear combinations of elementary functions $\left\{1_{[0, t]}, 0 \leq t \leq T\right\}$. Let the Hilbert space $\mathcal{H}$ be the closure of $\mathcal{E}$ with respect to the inner product

$$
\left\langle 1_{[0, t]}, 1_{[0, s]}\right\rangle_{\mathcal{H}_{*}}=\frac{\Gamma(1 / \alpha)}{2 \pi(\alpha-1)}\left((t+s)^{\frac{\alpha-1}{\alpha}}-|t-s|^{\frac{\alpha-1}{\alpha}}\right) .
$$

The map $1_{[0, t]} \mapsto W_{t}^{\alpha}$ is an isometry between $\mathcal{E}$ and the Gaussian space $W^{\alpha}(\varphi)$ of $\left\{W_{t}^{\alpha}, t \geq\right.$ $0\}$, which can be extended to $\mathcal{H}$. We denote the extension by

$$
\varphi \mapsto W^{\alpha}(\varphi)=\int_{0}^{t} \varphi(s) d W_{s}^{\alpha}
$$

Let $f \in C_{b}^{\infty}\left(\mathbb{R}^{n}\right), \varphi_{i} \in \mathcal{H}$, and let $\mathcal{S}$ denote the space of all smooth functionals of the following form:

$$
F=f\left(W^{\alpha}\left(\varphi_{1}\right), W^{\alpha}\left(\varphi_{2}\right), \ldots, W^{\alpha}\left(\varphi_{n}\right)\right)
$$

where $f \in C_{b}^{\infty}\left(\mathbb{R}^{n}\right)$ means $f$ and all their derivatives are bounded. The derivative operator $D^{\alpha}$ (the Malliavin derivative) of functionals $F$ of the above form is defined as

$$
D^{\alpha} F=\sum_{j=1}^{n} \frac{\partial f}{\partial x_{j}}\left(W^{\alpha}\left(\varphi_{1}\right), W^{\alpha}\left(\varphi_{2}\right), \ldots, W^{\alpha}\left(\varphi_{n}\right)\right) \varphi_{j}
$$

Then $D^{\alpha}$ is closable from $L^{2}(\Omega)$ into $L^{2}(\Omega ; \mathcal{H})$. Denote by $\mathbb{D}^{1,2}$ the closure of $\mathcal{S}$ endowed with the norm

$$
\left\|F_{t}\right\|_{1,2}:=\sqrt{E|F|^{2}+E\left\|D^{\alpha} F\right\|_{\mathcal{H}}^{2}}
$$

The divergence integral $\delta^{\alpha}$ is the adjoint of $D^{\alpha}$. Its domain is denoted by $\operatorname{Dom}\left(\delta^{\alpha}\right)$. We say that a random variable $u \in L^{2}(\Omega ; \mathcal{H})$ belongs to $\operatorname{Dom}\left(\delta^{\alpha}\right)$ if, for all $F \in \mathcal{S}$,

$$
E\left|\left\langle D^{\alpha} F, u\right\rangle_{\mathcal{H}}\right| \leq c\|F\|_{L^{2}(\Omega)}
$$

In these cases, for any $u \in \mathbb{D}^{1,2}, \delta^{\alpha}(u)$ is defined by

$$
E\left[F \delta^{\alpha}(u)\right]=E\left\langle D^{\alpha} F, u\right\rangle_{\mathcal{H}} .
$$

We have $\mathbb{D}^{1,2} \subset \operatorname{Dom}\left(\delta^{\alpha}\right)$. We will use the notations

$$
\delta^{\alpha}(u)=\int_{0}^{T} u_{s} d W_{s}^{\alpha}
$$

to express the Skorohod integral, and the indefinite Skorohod integral is defined as

$$
\int_{0}^{t} u_{s} d W_{s}^{\alpha}=\delta^{\alpha}\left(u 1_{[0, t]}\right)
$$




\section{The variation of temporal process}

Let $\{u(t, x), t \geq 0, x \in \mathbb{R}\}$ be the solution to the Cauchy problem (1.4). Then we have

$$
u(t, x)=\int_{0}^{t} \int_{\mathbb{R}} p_{\alpha}(t-s, x-y) B(d s, d y)
$$

where $p_{\alpha}(t, x)$ is the transition density function of a one-dimensional symmetry $\alpha$-stable Lévy process $X$ satisfying

$$
\int_{\mathbb{R}} p_{\alpha}(t, x) e^{i x \xi} d x=e^{-t|\xi|^{\alpha}}
$$

for $\xi \in \mathbb{R}$ and $t \geq 0$. In this section, we investigate the strong $p$-variation of the temporal process $W^{\alpha}=\left\{W_{t}^{\alpha}=u(t, \cdot), t \geq 0\right\}$.

Recall that a continuous process $X$ has a strong $p$-variation $(p>0)$ if

$$
u c p-\lim _{\varepsilon \downarrow 0} \frac{1}{\varepsilon} \int_{0}^{t}\left|X_{s+\varepsilon}-X_{s}\right|^{p} d s
$$

exists, where the notation ucp means the uniform convergence in probability on each compact interval. The limit is denoted by $[X, X]_{t}^{(p)}$ and is called the $p$-strong variation. If the $p$-strong variation exists, then for every $q>p>0,[X, X]_{t}^{(q)}=0$. When $p=2$ we call $[X, X]_{t}^{(p)}$ the quadratic covariation of $X$ and is denoted by $[X, X]$, i.e.

$$
[X, X]:=u c p-\lim _{\varepsilon \downarrow 0} \frac{1}{\varepsilon} \int_{0}^{t}\left(X_{s+\varepsilon}-X_{s}\right)^{2} d s
$$

For ucp-convergence we have the next perfect result due to Russo and Vallois [18].

Lemma 3.1 (Russo and Vallois [18]) Let $\left\{X^{\varepsilon}, \varepsilon>0\right\}$ be a set of continuous processes. We assume

- For any $\varepsilon>0$, the process $t \mapsto X_{t}^{\varepsilon}$ is increasing.

- There is a continuous process $X=\left(X_{t}, t \geq 0\right)$ such that $X_{t}^{\varepsilon} \rightarrow X_{t}$ in probability as $\varepsilon$ goes to zero.

Then $Z^{\varepsilon}$ converges to $X u c p$.

Theorem 3.1 Let $1<\alpha<2$. Then, for every $t>0$, we have

$$
\lim _{\varepsilon \downarrow 0} \frac{1}{\varepsilon} \int_{0}^{t}\left|W_{s+\varepsilon}^{\alpha}-W_{s}^{\alpha}\right|^{\frac{2 \alpha}{\alpha-1}} d s=\kappa_{\alpha} t
$$

in probability, i.e.

$$
\left[W^{\alpha}, W^{\alpha}\right]_{t}^{(p)}=\kappa_{\alpha} t
$$

with $p=\frac{2 \alpha}{\alpha-1}$, where $\lambda_{\alpha}=\left(\frac{\Gamma(1 / \alpha)}{\pi(\alpha-1)}\right) \frac{\alpha}{\alpha-1} E|\xi|^{\frac{2 \alpha}{\alpha-1}}$ with $\xi$ being a standard normal random variable. 
Proof Let $\varepsilon, t>0$ be given. Set

$$
\mathcal{C}^{\alpha}(t, \varepsilon):=\frac{1}{\varepsilon} \int_{0}^{t}\left|W_{s+\varepsilon}^{\alpha}-W_{s}^{\alpha}\right|^{\frac{2 \alpha}{\alpha-1}} d s
$$

By Lemma 3.1 one only needs to show that $\mathcal{C}^{\alpha}(t, \varepsilon)$ converges to $\lambda_{\alpha} t$ in $L^{2}(\Omega)$, as $\varepsilon \rightarrow 0$. Note that

$$
\begin{aligned}
E\left[\left(W_{s+\varepsilon}^{\alpha}-W_{s}^{\alpha}\right)^{2}\right] & =\kappa_{\alpha}\left((s+\varepsilon)^{\frac{\alpha-1}{\alpha}}-2^{\frac{1}{\alpha}}\left((s+\varepsilon+s)^{\frac{\alpha-1}{\alpha}}-\varepsilon^{\frac{\alpha-1}{\alpha}}\right)+s^{\frac{\alpha-1}{\alpha}}\right) \\
& =\kappa_{\alpha}(s+\varepsilon)^{\frac{\alpha-1}{\alpha}} f(x)
\end{aligned}
$$

for $x=\frac{\varepsilon}{s+\varepsilon}$ and $s>0$, by (2.3). It is clear that

$$
f(x)=1-2^{\frac{1}{\alpha}}\left((2-x)^{\frac{\alpha-1}{\alpha}}-x^{\frac{\alpha-1}{\alpha}}\right)+(1-x)^{\frac{\alpha-1}{\alpha}}=2^{\frac{1}{\alpha}} x^{\frac{\alpha-1}{\alpha}}+o\left(x^{\frac{\alpha-1}{\alpha}}\right)
$$

as $x \rightarrow 0$, and

$$
\lim _{\varepsilon \downarrow 0} \frac{1}{\varepsilon^{\frac{\alpha-1}{\alpha}}} E\left[\left(W_{s+\varepsilon}^{\alpha}-W_{s}^{\alpha}\right)^{2}\right]=\frac{\Gamma(1 / \alpha)}{\pi(\alpha-1)}
$$

for $s \geq \delta$. We have

$$
\begin{aligned}
\lim _{\varepsilon \downarrow 0} \frac{1}{\varepsilon} E\left[\left|W_{s+\varepsilon}^{\alpha}-W_{s}^{\alpha}\right|^{\frac{2 \alpha}{\alpha-1}}\right] & =\lim _{\varepsilon \downarrow 0} \frac{1}{\varepsilon} E|\xi|^{\frac{2 \alpha}{\alpha-1}}\left(E\left[\left(W_{s+\varepsilon}^{\alpha}-W_{s}^{\alpha}\right)^{2}\right]\right)^{\frac{\alpha}{\alpha-1}} \\
& =E|\xi|^{\frac{2 \alpha}{\alpha-1}}\left(\frac{\Gamma(1 / \alpha)}{\pi(\alpha-1)}\right)^{\frac{\alpha}{\alpha-1}}=\lambda_{\alpha}
\end{aligned}
$$

for $s>0$ and

$$
\lim _{\varepsilon \downarrow 0} E\left(\mathcal{C}^{\alpha}(t, \varepsilon)\right)=\lambda_{\alpha} t
$$

Thus, to obtain the result it suffices to establish that

$$
\lim _{\varepsilon \downarrow 0} E\left(\mathcal{C}^{\alpha}(t, \varepsilon)\right)^{2}=\left(\lambda_{\alpha}\right)^{2} t^{2}
$$

for all $t>0$. We get

$$
E\left(\mathcal{C}^{\alpha}(t, \varepsilon)\right)^{2}=\frac{2}{\varepsilon^{2}} \int_{0}^{t} d s \int_{0}^{s} f_{\varepsilon}(s, r) d r
$$

where

$$
f_{\varepsilon}(s, r)=E\left|\left(W_{s+\varepsilon}^{\alpha}-W_{s}^{\alpha}\right)\left(W_{r+\varepsilon}^{\alpha}-W_{r}^{\alpha}\right)\right|^{\frac{2 \alpha}{\alpha-1}} .
$$

Recall that if $\left(B_{1}, B_{2}\right)$ is a Gaussian couple, then we can write

$$
B_{2}=\frac{\operatorname{Cov}\left(B_{1}, B_{2}\right)}{\operatorname{Var}\left(B_{1}\right)} G_{1}+\sqrt{\operatorname{Var}\left(B_{2}\right)-\frac{\operatorname{Cov}^{2}\left(B_{1}, B_{2}\right)}{\operatorname{Var}\left(B_{1}\right)}} \eta,
$$


where $\eta$ is a standard normal random variable independent of $B_{1}$ and $\operatorname{Var}(\cdot)$ denotes the variance. Let $B_{1}=W_{s+\varepsilon}^{\alpha}-W_{s}^{\alpha}, B_{2}=W_{r+\varepsilon}^{\alpha}-W_{r}^{\alpha}, \mu_{\varepsilon}(s, r)=E B_{1} B_{2}$, and

$$
\Upsilon_{\varepsilon}(s, r)=\operatorname{Var}\left(B_{1}\right) \operatorname{Var}\left(B_{2}\right)-\mu_{\varepsilon}(s, r)^{2} .
$$

We get

$$
\begin{aligned}
\frac{1}{\varepsilon^{2}} f_{\varepsilon}(s, r) & =\frac{1}{\varepsilon^{2}} E\left|B_{1} B_{2}\right|^{\frac{2 \alpha}{\alpha-1}} \\
& =E\left(\left|\frac{B_{1}}{\sqrt{\operatorname{Var}\left(B_{1}\right)}}\right|^{\frac{2 \alpha}{\alpha-1}}\left|\frac{\mu_{\varepsilon}(s, r)}{\varepsilon^{1+b}} \frac{B_{1}}{\sqrt{\operatorname{Var}\left(B_{1}\right)}}+\frac{1}{\varepsilon^{\frac{\alpha}{\alpha-1}}} \sqrt{\Upsilon_{\varepsilon}(s, r)} \eta\right|^{\frac{2 \alpha}{\alpha-1}}\right) \\
& =E\left(|\zeta|^{\frac{2 \alpha}{\alpha-1}}\left|\frac{\mu_{\varepsilon}(s, r)}{\varepsilon^{\frac{\alpha}{\alpha-1}}} \zeta+\frac{1}{\varepsilon^{\frac{\alpha}{\alpha-1}}} \sqrt{\Upsilon_{\varepsilon}(s, r)} \eta\right|^{\frac{2 \alpha}{\alpha-1}}\right)
\end{aligned}
$$

with a standard normal random variable $\zeta$ independent of $\eta$.

By Lemma 2.1, it is obvious that

$$
\frac{1}{\varepsilon^{\frac{\alpha}{\alpha-1}}} \sqrt{\Upsilon_{\varepsilon}(s, r)} \leq \frac{1}{\varepsilon^{\frac{\alpha}{\alpha-1}}} \sqrt{E\left(W_{s+\varepsilon}^{\alpha}-W_{s}^{\alpha}\right)^{2} E\left(W_{r+\varepsilon}^{\alpha}-W_{r}^{\alpha}\right)^{2}} \asymp C .
$$

Combining this with (3.5) and Lemma 2.3, we have

$$
\int_{0}^{\varepsilon} d s \int_{0}^{s} \frac{1}{\varepsilon^{2}} f_{\varepsilon}(s, r) d r, \int_{\varepsilon}^{t} d s \int_{s-\varepsilon}^{s} \frac{1}{\varepsilon^{2}} f_{\varepsilon}(s, r) d r \longrightarrow 0
$$

as $\varepsilon \rightarrow 0$. By (3.3) and (3.5) it follows that

$$
\begin{aligned}
E\left(\mathcal{C}^{\alpha}(t, \varepsilon)\right)^{2}= & \frac{2}{\varepsilon^{2}} \int_{0}^{t} d s \int_{0}^{s} f_{\varepsilon}(s, r) d r \\
= & \frac{2}{\varepsilon^{2}} \int_{\varepsilon}^{t} d s \int_{0}^{s-\varepsilon} f_{\varepsilon}(s, r) d r+\frac{2}{\varepsilon^{2}} \int_{\varepsilon}^{t} d s \int_{s-\varepsilon}^{s} f_{\varepsilon}(s, r) d r \\
& +\frac{2}{\varepsilon^{2}} \int_{0}^{\varepsilon} d s \int_{0}^{s} f_{\varepsilon}(s, r) d r \\
\longrightarrow & 2 \int_{0}^{t} d s \int_{0}^{s}\left(\lambda_{\alpha}\right)^{2} d r=\left(\lambda_{\alpha}\right)^{2} t^{2}
\end{aligned}
$$

as $\varepsilon \rightarrow 0$, by Lebesgue's dominated convergence theorem, the theorem follows.

From Yan et al. [19] and the above theorem, we can naturally introduce the next definition.

Definition 3.1 For all $t \geq 0$, define the integral

$$
I_{\varepsilon}(f, t)=\frac{1}{\varepsilon^{\frac{\alpha-1}{\alpha}}} \int_{0}^{t}\left\{f\left(W_{s+\varepsilon}^{\alpha}\right)-f\left(W_{s}^{\alpha}\right)\right\}\left(W_{s+\varepsilon}^{\alpha}-W_{s}^{\alpha}\right) d s^{\frac{\alpha-1}{\alpha}},
$$

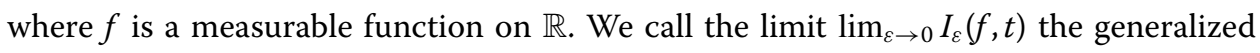
quadratic covariation of $f\left(W^{\alpha}\right)$ and $W^{\alpha}$, denoted by $\left\langle f\left(W^{\alpha}\right), W^{\alpha}\right\rangle^{(\alpha)}$, provided this limit exists in probability. 
Proposition 3.1 For all $1<\alpha<2$ and $t \geq 0$, we have

$$
\left\langle B^{H}, B^{H}\right\rangle_{t}^{(H)}=\frac{\Gamma(1 / \alpha)}{\pi(\alpha-1)} t^{\frac{\alpha-1}{\alpha}} .
$$

Moreover, for every $f \in C^{1}(\mathbb{R})$, we have

$$
\left\langle f\left(W^{\alpha}\right),\left.W^{\alpha}\right|_{t} ^{(\alpha)}=\frac{\Gamma(1 / \alpha)}{\pi(\alpha-1)} \int_{0}^{t} f^{\prime}\left(W_{s}^{\alpha}\right) d s^{\frac{\alpha-1}{\alpha}} .\right.
$$

Proof It is suffices to estimate

$$
\begin{aligned}
& E\left|\int_{0}^{t}\left(W_{s+\varepsilon}^{\alpha}-W_{s}^{\alpha}\right)^{2} d s^{\frac{\alpha-1}{\alpha}}-\frac{\Gamma(1 / \alpha)}{\pi(\alpha-1)}(t \varepsilon)^{\frac{\alpha-1}{\alpha}}\right|^{2} \\
& \quad=E\left|\int_{0}^{t}\left(\left(W_{s+\varepsilon}^{\alpha}-W_{s}^{\alpha}\right)^{2}-\frac{\Gamma(1 / \alpha)}{\pi(\alpha-1)} \varepsilon^{\frac{\alpha-1}{\alpha}}\right) d s^{\frac{\alpha-1}{\alpha}}\right|^{2} \\
& \quad \equiv \int_{0}^{t} \int_{0}^{t} X_{\varepsilon}(s, r) d s^{\frac{\alpha-1}{\alpha}} d r^{\frac{\alpha-1}{\alpha}}
\end{aligned}
$$

for all $\varepsilon>0$, where

$$
\begin{aligned}
X_{\varepsilon}(y, z)= & E\left(\left(W_{s+\varepsilon}^{\alpha}-W_{s}^{\alpha}\right)^{2}-\frac{\Gamma(1 / \alpha)}{\pi(\alpha-1)} \varepsilon^{\frac{\alpha-1}{\alpha}}\right)\left(\left(W_{r+\varepsilon}^{\alpha}-W_{r}^{\alpha}\right)^{2}-\frac{\Gamma(1 / \alpha)}{\pi(\alpha-1)} \varepsilon^{\frac{\alpha-1}{\alpha}}\right) \\
= & E\left(\left(W_{s+\varepsilon}^{\alpha}-W_{s}^{\alpha}\right)^{2}\left(W_{r+\varepsilon}^{\alpha}-W_{r}^{\alpha}\right)^{2}\right) \\
& -\frac{\Gamma(1 / \alpha)}{\pi(\alpha-1)} \varepsilon^{\frac{\alpha-1}{\alpha}}\left(E\left(W_{s+\varepsilon}^{\alpha}-W_{s}^{\alpha}\right)^{2}+E\left(W_{r+\varepsilon}^{\alpha}-W_{r}^{\alpha}\right)^{2}\right)+\left(\frac{\Gamma(1 / \alpha)}{\pi(\alpha-1)} \varepsilon^{\frac{\alpha-1}{\alpha}}\right)^{2} \\
= & E\left(\left(W_{s+\varepsilon}^{\alpha}-W_{s}^{\alpha}\right)^{2}\left(W_{r+\varepsilon}^{\alpha}-W_{r}^{\alpha}\right)^{2}\right)-\left(\frac{\Gamma(1 / \alpha)}{\pi(\alpha-1)} \varepsilon^{\frac{\alpha-1}{\alpha}}\right)^{2}
\end{aligned}
$$

for every $t>0$ and $y, z \in \mathbb{R}$. By an elementary calculation we can show that

$$
\begin{aligned}
E & \left(\left(W_{s+\varepsilon}^{\alpha}-W_{s}^{\alpha}\right)^{2}\left(W_{r+\varepsilon}^{\alpha}-W_{r}^{\alpha}\right)^{2}\right) \\
= & E\left(W_{s+\varepsilon}^{\alpha}-W_{s}^{\alpha}\right)^{2} E\left(W_{r+\varepsilon}^{\alpha}-W_{r}^{\alpha}\right)^{2} \\
& +2\left[E\left(W_{s+\varepsilon}^{\alpha}-W_{s}^{\alpha}\right)\left(W_{r+\varepsilon}^{\alpha}-W_{r}^{\alpha}\right)\right]^{2} \\
= & \left(\frac{\Gamma(1 / \alpha)}{\pi(\alpha-1)} \varepsilon^{\frac{\alpha-1}{\alpha}}\right)^{2}+2\left[E\left(W_{s+\varepsilon}^{\alpha}-W_{s}^{\alpha}\right)\left(W_{r+\varepsilon}^{\alpha}-W_{r}^{\alpha}\right)\right]^{2}
\end{aligned}
$$

for all $\varepsilon>0$ and $y, z \in I_{x}$, which implies

$$
X_{\varepsilon}(y, z)=2\left[E\left(W_{s+\varepsilon}^{\alpha}-W_{s}^{\alpha}\right)\left(W_{r+\varepsilon}^{\alpha}-W_{r}^{\alpha}\right)\right]^{2} .
$$

It follows from Lemma 2.1, Lemma 2.3, and the fact

$$
\left|E\left(W_{s+\varepsilon}^{\alpha}-W_{s}^{\alpha}\right)\left(W_{r+\varepsilon}^{\alpha}-W_{r}^{\alpha}\right)\right| \leq C \varepsilon^{\frac{\alpha-1}{\alpha}}
$$


for $|s-r|<\varepsilon$ that

$$
\int_{0}^{t} \int_{0}^{t} X_{\varepsilon}(s, r) d s^{\frac{\alpha-1}{\alpha}} d r^{\frac{\alpha-1}{\alpha}}=O\left(\varepsilon^{\beta+\frac{2(\alpha-1)}{\alpha}}\right) \quad(\varepsilon \downarrow 0)
$$

for some $\beta>0$, which gives

$$
\left\langle W^{\alpha}, W^{\alpha}\right\rangle_{t}^{(\alpha)}=\frac{\Gamma(1 / \alpha)}{\pi(\alpha-1)} t^{\frac{\alpha-1}{\alpha}}
$$

for all $t \geq 0$.

On the other hand, by Hölder continuity of $W^{\alpha}$ we get

$$
\lim _{\varepsilon \downarrow 0} \frac{1}{\varepsilon^{\frac{\alpha-1}{\alpha}}} \int_{0}^{t} o\left(W_{s+\varepsilon}^{\alpha}-W_{s}^{\alpha}\right)\left(W_{s+\varepsilon}^{\alpha}-W_{s}^{\alpha}\right)^{2} d s^{\frac{\alpha-1}{\alpha}}=0
$$

almost surely. It follows that

$$
\begin{aligned}
& \lim _{\varepsilon \downarrow 0} \frac{1}{\varepsilon^{\frac{\alpha-1}{\alpha}}} \int_{0}^{t}\left\{f\left(W_{s+\varepsilon}^{\alpha}\right)-f\left(W_{s}^{\alpha}\right)\right\}\left(W_{s+\varepsilon}^{\alpha}-W_{s}^{\alpha}\right) d s^{\frac{\alpha-1}{\alpha}} \\
& \quad=\lim _{\varepsilon \downarrow 0} \frac{1}{\varepsilon^{\frac{\alpha-1}{\alpha}}} \int_{0}^{t} f^{\prime}\left(W_{s}^{\alpha}\right)\left(W_{s+\varepsilon}^{\alpha}-W_{s}^{\alpha}\right)^{2} d s^{\frac{\alpha-1}{\alpha}}=\frac{\Gamma(1 / \alpha)}{\pi(\alpha-1)} \int_{0}^{t} f^{\prime}\left(W_{s}^{\alpha}\right) d s^{\frac{\alpha-1}{\alpha}}
\end{aligned}
$$

almost surely. By the next lemma and the proposition the result follows.

Recall that the local Hölder index $\gamma_{0}$ of a continuous paths process $\left\{X_{t}: t \geq 0\right\}$ is the supremum of the exponents $\gamma$ verifying, for any $T>0$,

$$
P\left(\left\{\omega: \exists L(\omega)>0, \forall s, t \in[0, T],\left|X_{t}(\omega)-X_{s}(\omega)\right| \leq L(\omega)|t-s|^{\gamma}\right\}\right)=1 .
$$

Recently, Gradinaru-Nourdin [20] introduced the following very useful result.

Lemma 3.2 Assume that $f: \mathbb{R} \rightarrow \mathbb{R}$ is a function such that for all $x, y \in \mathbb{R}$,

$$
|f(x)-f(y)| \leq C|x-y|^{a}\left(1+x^{2}+y^{2}\right)^{b} \quad(C>0,0<a \leq 1, b>0) .
$$

Let $X$ be a locally Hölder continuous paths process with index $\gamma \in(0,1)$. Assume that $V$ is a bounded variation continuous paths process. For $t \geq 0, \varepsilon>0$, set

$$
X_{\varepsilon}^{f}(t)=\int_{0}^{t} f\left(\frac{X_{s+\varepsilon}-X_{s}}{\varepsilon^{\gamma}}\right) d s .
$$

If for each $t \geq 0$,

$$
\lim _{\varepsilon \rightarrow 0}=\left\|X_{\varepsilon}^{f}(t)-V_{t}\right\|_{L^{2}}^{2}=O\left(\varepsilon^{\alpha}\right)
$$

with $\alpha>0$, then for any $t \geq 0, \lim _{\varepsilon \rightarrow 0} X_{\varepsilon}^{f}(t)=V_{t}$ almost surely, and iff is non-negative, for any continuous stochastic process $\left\{Y_{t}: t \geq 0\right\}$,

$$
\lim _{\varepsilon \rightarrow 0} \int_{0}^{t} Y_{s} f\left(\frac{X_{s+\varepsilon}-X_{s}}{\varepsilon^{\gamma}}\right) d s \rightarrow \int_{0}^{t} Y_{s} d V_{s}
$$

almost surely, uniformly in t on each compact interval. 


\section{The existence of the generalized quadratic covariation}

In this section, we consider the existence of the generalized quadratic covariation of $f\left(W^{\alpha}\right)$ and $W^{\alpha}$, we do not need that $f$ is a $C^{1}$-function. The main idea is from Yan et al. [19]. Let $v_{\alpha}=\frac{\Gamma(1 / \alpha)}{\pi(\alpha-1)}$ and $\beta=\frac{\alpha-1}{\alpha}$. For $\varepsilon>0$, we consider the following decomposition:

$$
\begin{aligned}
& \frac{1}{\varepsilon^{\beta}} \int_{0}^{t}\left\{f\left(W_{s+\varepsilon}^{\alpha}\right)-f\left(W_{s}^{\alpha}\right)\right\}\left(W_{s+\varepsilon}^{\alpha}-W_{s}^{\alpha}\right) d s^{\beta} \\
& \quad=\frac{1}{\varepsilon^{\beta}} \int_{0}^{t} f\left(W_{s+\varepsilon}^{\alpha}\right)\left(W_{s+\varepsilon}^{\alpha}-W_{s}^{\alpha}\right) d s^{\beta}-\frac{1}{\varepsilon^{\beta}} \int_{0}^{t} f\left(W_{s}^{\alpha}\right)\left(W_{s+\varepsilon}^{\alpha}-W_{s}^{\alpha}\right) d s^{\beta} \\
& \quad \equiv I_{\varepsilon}^{+}(f, t)-I_{\varepsilon}^{-}(f, t)
\end{aligned}
$$

and define a set

$$
\mathscr{H}=\left\{g: \mathrm{g} \text { is a Borel function on } \mathbb{R} \text { such that }\|g\|_{\mathscr{H}}<\infty\right\},
$$

where

$$
\|g\|_{\mathscr{H}}^{2}:=\int_{0}^{T} \int_{\mathbb{R}}|g(x)|^{2} e^{-\frac{x^{2}}{2 v_{\alpha} s^{\beta}}} \frac{d x d s}{\sqrt{2 \pi v_{\alpha}} s^{1-\frac{\beta}{2}}}=E\left(\int_{0}^{T}\left|g\left(W_{s}^{\alpha}\right)\right|^{2} d s^{\beta}\right) .
$$

Then $\mathscr{H}=L^{2}(\mathbb{R}, \mu(d x))$, where

$$
\mu(d x)=\left(\int_{0}^{T} e^{-\frac{x^{2}}{2 v_{\alpha} s^{\beta}}} \frac{d s}{\sqrt{2 \pi v_{\alpha}} s^{1-\frac{\beta}{2}}}\right) d x
$$

and $\mu(\mathbb{R})=T^{2 H}<\infty$, which indicates that the set

$$
\mathscr{E}:=\left\{\text { all the elementary functions of the form } g_{\Delta}(x)=\sum_{i} g_{i} 1_{\left(x_{i-1}, x_{i}\right]}(x)\right\}
$$

is dense in $\mathscr{H}$, where $\left\{x_{i}, 0 \leq i \leq l\right\}$ is an finite sequence of real numbers satisfying $x_{i}<x_{i+1}$. In order to get the existence of the generalized quadratic covariation, we need first to present the following two statements:

(i) For $t \in[0,1]$ and any $\varepsilon>0, f \in C_{0}^{\infty} \cap \mathscr{H}, I_{\varepsilon}^{ \pm}(f, t) \in L^{2}(\Omega)$. That is,

$$
\begin{aligned}
& E\left|I_{\varepsilon}^{-}(f, t)\right|^{2} \leq C\|f\|_{\mathscr{H}}^{2}, \\
& E\left|I_{\varepsilon}^{+}(f, t)\right|^{2} \leq C\|f\|_{\mathscr{H}}^{2} .
\end{aligned}
$$

(ii) For every $f \in C_{0}^{\infty} \cap \mathscr{H}$ and $t \in[0,1], I_{\varepsilon}^{+}(f, t)$ and $I_{\varepsilon}^{-}(f, t)$ are Cauchy sequences in $L^{2}(\Omega)$. That is, for all $t \in[0,1]$

$$
\begin{aligned}
& E\left|I_{\varepsilon_{1}}^{-}(f, t)-I_{\varepsilon_{2}}^{-}(f, t)\right|^{2} \longrightarrow 0 \\
& E\left|I_{\varepsilon_{1}}^{+}(f, t)-I_{\varepsilon_{2}}^{+}(f, t)\right|^{2} \longrightarrow 0
\end{aligned}
$$


We divide the proof of the two statements into several parts which is similar to Yan et al. [19]. For simplicity, let $T=1$. We need the next elementary lemmas. Let $\varphi(x, y)$ denote the density function of $\left(W_{s}^{\alpha}, W_{r}^{\alpha}\right)(s>r>0)$. That is

$$
\varphi(x, y)=\frac{1}{2 \pi \rho} \exp \left\{-\frac{1}{2 \rho^{2}}\left(r^{\beta} x^{2}-2 \mu x y+s^{\beta} y^{2}\right)\right\}
$$

where $\mu=E\left(W_{s}^{\alpha} W_{r}^{\alpha}\right)$ and $\rho^{2}=\left(\kappa_{\alpha}\right)^{2} r^{\beta} s^{\beta}-\mu^{2}$.

Lemma 4.1 Let $f \in C^{1}(\mathbb{R})$ admit a compact support. Then we have

$$
\begin{aligned}
& \left|E\left[f^{\prime}\left(W_{s}^{\alpha}\right) f^{\prime}\left(W_{r}^{\alpha}\right)\right]\right| \leq \frac{C s^{\beta / 2}}{r^{\beta / 2}(s-r)^{\beta}}\left(E\left[\left|f\left(W_{s}^{\alpha}\right)\right|^{2}\right] E\left[\left|f\left(W_{r}^{\alpha}\right)\right|^{2}\right]\right)^{1 / 2}, \\
& \left|E\left[f^{\prime \prime}\left(W_{s}^{\alpha}\right) f\left(W_{r}^{\alpha}\right)\right]\right| \leq \frac{C}{(s-r)^{\beta}}\left(E\left[\left|f\left(W_{s}^{\alpha}\right)\right|^{2}\right] E\left[\left|f\left(W_{r}^{\alpha}\right)\right|^{2}\right]\right)^{1 / 2}
\end{aligned}
$$

for all $s>r>0$.

Proof By using an elementary calculation it follows that

$$
\begin{aligned}
& \int_{\mathbb{R}^{2}} f^{2}(y)\left(x-\frac{\mu}{\kappa_{\alpha} r^{\beta}} y\right)^{2} \varphi(x, y) d x d y \\
& \quad=\frac{\rho^{2}}{\kappa_{\alpha} r^{\beta}} \int_{\mathbb{R}} f^{2}(y) \frac{1}{\sqrt{2 \pi \kappa_{\alpha}} r^{\beta / 2}} e^{-\frac{y^{2}}{2 \kappa_{\alpha} r^{\beta}}} d y=\frac{\rho^{2}}{\kappa_{\alpha} r^{\beta}} E\left[\left|f\left(W_{r}^{\alpha}\right)\right|^{2}\right],
\end{aligned}
$$

which implies that

$$
\begin{aligned}
& \frac{1}{\rho^{4}} \int_{\mathbb{R}^{2}}\left|f(x) f(y)\left(\kappa_{\alpha} s^{\beta} y-\mu x\right)\left(\kappa_{\alpha} r^{\beta} x-\mu y\right)\right| \varphi(x, y) d x d y \\
& \quad \leq \frac{\kappa_{\alpha}(r s)^{\beta / 2}}{\rho^{2}}\left(E\left[\left|f\left(W_{s}^{\alpha}\right)\right|^{2}\right] E\left[\left|f\left(W_{r}^{\alpha}\right)\right|^{2}\right]\right)^{1 / 2} \\
& \quad \leq \frac{C s^{\beta / 2}}{r^{\beta / 2}(s-r)^{\beta}}\left(E\left[\left|f\left(W_{s}^{\alpha}\right)\right|^{2}\right] E\left[\left|f\left(W_{r}^{\alpha}\right)\right|^{2}\right]\right)^{1 / 2}
\end{aligned}
$$

by Lemma 2.4, we have

$$
\begin{aligned}
\left|E\left[f^{\prime}\left(W_{s}^{\alpha}\right) f^{\prime}\left(W_{r}^{\alpha}\right)\right]\right| & =\left|\int_{\mathbb{R}^{2}} f(x) f(y) \frac{\partial^{2}}{\partial x \partial y} \varphi(x, y) d x d y\right| \\
& =\left|\int_{\mathbb{R}^{2}} f(x) f(y)\left\{\frac{1}{\rho^{4}}\left(\kappa_{\alpha} s^{\beta} y-\mu x\right)\left(\kappa_{\alpha} r^{\beta} x-\mu y\right)+\frac{\mu}{\rho^{2}}\right\} \varphi(x, y) d x d y\right| \\
& \leq \frac{C s^{\beta / 2}}{r^{\beta / 2}(s-r)^{\beta}}\left(E\left[\left|f\left(W_{s}^{\alpha}\right)\right|^{2}\right] E\left[\left|f\left(W_{r}^{\alpha}\right)\right|^{2}\right]\right)^{1 / 2} .
\end{aligned}
$$

This gives the first estimate, by a similarly argument, one can obtain the second estimate.

Proof of the statement (i) Let $f \in C_{0}^{\infty}$. Noting that for all $\varepsilon>0$ and $t \geq 0$, we have

$$
E\left|I_{\varepsilon}^{-}(f, t)\right|^{2}=\frac{1}{\varepsilon^{2 \beta}} \int_{0}^{t} \int_{0}^{t} E\left[f\left(W_{s}^{\alpha}\right) f\left(W_{r}^{\alpha}\right)\left(W_{s+\varepsilon}^{\alpha}-W_{s}^{\alpha}\right)\left(W_{r+\varepsilon}^{\alpha}-W_{r}^{\alpha}\right)\right] d s^{\beta} d r^{\beta} .
$$


For all $s, r>0$ and $\varepsilon>0$, let us estimate the expression

$$
\Lambda_{\varepsilon}(s, r):=E\left[f\left(W_{s}^{\alpha}\right) f\left(W_{r}^{\alpha}\right)\left(W_{s+\varepsilon}^{\alpha}-W_{s}^{\alpha}\right)\left(W_{r+\varepsilon}^{\alpha}-W_{r}^{\alpha}\right)\right] .
$$

Note that

$$
\begin{aligned}
\Lambda \varepsilon(s, r)= & E\left[f\left(W_{s}^{\alpha}\right) f\left(W_{r}^{\alpha}\right)\left(W_{s+\varepsilon}^{\alpha}-W_{s}^{\alpha}\right) \int_{r}^{r+\varepsilon} d W_{l}^{\alpha}\right] \\
= & E\left[D^{\alpha} f\left(W_{s}^{\alpha}\right) f\left(W_{r}^{\alpha}\right)\left(W_{s+\varepsilon}^{\alpha}-W_{s}^{\alpha}\right), 1_{[r, r+\varepsilon]}\right\rangle_{\mathcal{H}} \\
= & E\left[W_{s}^{\alpha}\left(W_{r+\varepsilon}^{\alpha}-W_{r}^{\alpha}\right)\right] E\left[f^{\prime}\left(W_{s}^{\alpha}\right) f\left(W_{r}^{\alpha}\right)\left(W_{s+\varepsilon}^{\alpha}-W_{s}^{\alpha}\right)\right] \\
& +E\left[W_{r}^{\alpha}\left(W_{r+\varepsilon}^{\alpha}-W_{r}^{\alpha}\right)\right] E\left[f\left(W_{s}^{\alpha}\right) f^{\prime}\left(W_{r}^{\alpha}\right)\left(W_{s+\varepsilon}^{\alpha}-W_{s}^{\alpha}\right)\right] \\
& +E\left[\left(W_{r+\varepsilon}^{\alpha}-W_{r}^{\alpha}\right)\left(W_{s+\varepsilon}^{\alpha}-W_{s}^{\alpha}\right)\right] E\left[f\left(B_{s}^{H}\right) f\left(B_{r}^{H}\right)\right] \\
= & E\left[W_{s}^{\alpha}\left(W_{r+\varepsilon}^{\alpha}-W_{r}^{\alpha}\right)\right] E\left[W_{s}^{\alpha}\left(W_{s+\varepsilon}^{\alpha}-W_{s}^{\alpha}\right)\right] E\left[f^{\prime \prime}\left(W_{s}^{\alpha}\right) f\left(W_{r}^{\alpha}\right)\right] \\
& +E\left[W_{s}^{\alpha}\left(W_{r+\varepsilon}^{\alpha}-W_{r}^{\alpha}\right)\right] E\left[W_{r}^{\alpha}\left(W_{s+\varepsilon}^{\alpha}-W_{s}^{\alpha}\right)\right] E\left[f^{\prime}\left(W_{s}^{\alpha}\right) f^{\prime}\left(W_{r}^{\alpha}\right)\right] \\
& +E\left[W_{r}^{\alpha}\left(W_{r+\varepsilon}^{\alpha}-W_{r}^{\alpha}\right)\right] E\left[W_{s}^{\alpha}\left(W_{s+\varepsilon}^{\alpha}-W_{s}^{\alpha}\right)\right] E\left[f^{\prime}\left(W_{s}^{\alpha}\right) f^{\prime}\left(W_{r}^{\alpha}\right)\right] \\
& +E\left[W_{r}^{\alpha}\left(W_{r+\varepsilon}^{\alpha}-W_{r}^{\alpha}\right)\right] E\left[W_{r}^{\alpha}\left(W_{s+\varepsilon}^{\alpha}-W_{s}^{\alpha}\right)\right] E\left[f\left(W_{s}^{\alpha}\right) f^{\prime \prime}\left(W_{r}^{\alpha}\right)\right] \\
& +E\left[\left(W_{r+\varepsilon}^{\alpha}-W_{r}^{\alpha}\right)\left(W_{s+\varepsilon}^{\alpha}-W_{s}^{\alpha}\right)\right] E\left[f\left(B_{s}^{H}\right) f\left(B_{r}^{H}\right)\right] \\
& 5 \\
\equiv & \sum_{j=1} \Psi_{\varepsilon}(s, r, j) .
\end{aligned}
$$

By Cauchy's inequality, it is easy to see that, for $|s-r|<\varepsilon \leq 1$,

$$
\left|E\left[\left(W_{r+\varepsilon}^{\alpha}-W_{r}^{\alpha}\right)\left(W_{s+\varepsilon}^{\alpha}-W_{s}^{\alpha}\right)\right]\right| \leq C \varepsilon^{\beta} \leq C \frac{\varepsilon^{2 \beta}}{|s-r|^{\beta}}
$$

It follows from Cauchy's inequality, Lemma 2.3, and the fact

$$
\begin{aligned}
E\left[f^{2}\left(W_{r}^{\alpha}\right)\right] & =\int_{\mathbb{R}} f^{2}(x) \frac{1}{\sqrt{2 \pi v_{\alpha}} r^{\beta / 2}} e^{-\frac{x^{2}}{2 v_{\alpha} r^{\beta}}} d x \\
& \leq \frac{s^{\beta / 2}}{r^{\beta / 2}} \int_{\mathbb{R}} f^{2}(x) \frac{1}{\sqrt{2 \pi v_{\alpha}} s^{\beta / 2}} e^{-\frac{x^{2}}{2 v_{\alpha} s^{\beta}}} d x=\frac{s^{\beta / 2}}{r^{\beta / 2}} E\left[f^{2}\left(W_{s}^{\alpha}\right)\right],
\end{aligned}
$$

we have

$$
\frac{1}{\varepsilon^{2 \beta}}\left|\int_{0}^{t} \int_{0}^{t} \Psi_{\varepsilon}(s, r, 5) d s^{\beta} d r^{\beta}\right| \leq C\|f\|_{\mathscr{H}}^{2}
$$

for all $0<\varepsilon \leq 1$.

Combing Lemma 4.1, Lemma 2.2, Lemma 2.3, and (4.6) we get

$$
\begin{aligned}
& \frac{1}{\varepsilon^{2 \beta}}\left|\int_{0}^{t} \int_{0}^{t} \Psi_{\varepsilon}(s, r, 1) d s^{\beta} d r^{\beta}\right| \\
& \quad \leq \int_{0}^{t} \int_{0}^{t}\left|E\left[f^{\prime \prime}\left(W_{s}^{\alpha}\right) f\left(W_{r}^{\alpha}\right)\right]\right| d r^{\beta} d s^{\beta}
\end{aligned}
$$




$$
\begin{aligned}
& \leq C \int_{0}^{t} \int_{0}^{s} \frac{1}{(s-r)^{\beta}} E\left|f\left(W_{s}^{\alpha}\right) f\left(W_{r}^{\alpha}\right)\right| d r^{\beta} d s^{\beta} \\
& \leq C \int_{0}^{t} E\left[f^{2}\left(W_{s}^{\alpha}\right)\right] s^{\frac{5}{4} \beta-1} d s \int_{0}^{s} \frac{s^{\beta / 4}}{(s-r)^{\beta} r^{1-\frac{3}{4} \beta}} d r \leq C\|f\|_{\mathscr{H}}^{2}
\end{aligned}
$$

for all $\varepsilon>0$ and $t \geq 0$. In a similar way, we can estimate

$$
\frac{1}{\varepsilon^{2 \beta}}\left|\int_{0}^{t} \int_{0}^{t} \Psi_{\varepsilon}(s, r, j) d s^{\beta} d r^{\beta}\right|
$$

for $j \in\{2,3,4\}$. Thus, we have given the estimate (4.2). In the same way, one finds (4.3).

Proof of the statement (ii) By the first statement we can give the second statement with $f \in C_{0}^{\infty}$. In fact, for all $\varepsilon_{1}, \varepsilon_{2}>0$ and $t \geq 0$, we have

$$
\begin{aligned}
E\left|I_{\varepsilon_{1}}^{-}(f, t)-I_{\varepsilon_{2}}^{-}(f, t)\right|^{2}= & \frac{1}{\varepsilon_{1}^{2 \beta}} \int_{0}^{t} \int_{0}^{t} E f\left(W_{s}^{\alpha}\right) f\left(W_{r}^{\alpha}\right) \\
& \cdot\left(W_{s+\varepsilon_{1}}^{\alpha}-W_{s}^{\alpha}\right)\left(W_{r+\varepsilon_{1}}^{\alpha}-W_{r}^{\alpha}\right) d r^{\beta} d s^{\beta} \\
& -\frac{2}{\varepsilon_{1}^{\beta} \varepsilon_{2}^{\beta}} \int_{0}^{t} \int_{0}^{t} E f\left(W_{s}^{\alpha}\right) f\left(W_{r}^{\alpha}\right) \\
& \cdot\left(W_{s+\varepsilon_{1}}^{\alpha}-W_{s}^{\alpha}\right)\left(W_{r+\varepsilon_{2}}^{\alpha}-W_{r}^{\alpha}\right) d r^{\beta} d s^{\beta} \\
& +\frac{1}{\varepsilon_{2}^{2 \beta}} \int_{0}^{t} \int_{0}^{t} E f\left(W_{r}^{\alpha}\right) f\left(W_{r}^{\alpha}\right) \\
& \cdot\left(W_{s+\varepsilon_{2}}^{\alpha}-W_{s}^{\alpha}\right)\left(W_{r+\varepsilon_{2}}^{\alpha}-W_{r}^{\alpha}\right) d r^{\beta} d s^{\beta} .
\end{aligned}
$$

Set

$$
\Phi_{s, r}(1, \varepsilon)=E\left[f\left(W_{s}^{\alpha}\right) f\left(W_{r}^{\alpha}\right)\left(W_{s+\varepsilon}^{\alpha}-W_{s}^{\alpha}\right)\left(W_{r+\varepsilon}^{\alpha}-W_{r}^{\alpha}\right)\right]
$$

and

$$
\Phi_{s, r}\left(2, \varepsilon_{1}, \varepsilon_{2}\right)=E\left[f\left(W_{s}^{\alpha}\right) f\left(W_{r}^{\alpha}\right)\left(W_{s+\varepsilon_{1}}^{\alpha}-W_{s}^{\alpha}\right)\left(W_{r+\varepsilon_{2}}^{\alpha}-W_{r}^{\alpha}\right)\right]
$$

for all $s, r \geq 0$ and $\varepsilon_{1}, \varepsilon_{2}, \varepsilon>0$. Then we have

$$
\begin{aligned}
& E\left|I_{\varepsilon_{1}}^{-}(f, t)-I_{\varepsilon_{2}}^{-}(f, t)\right|^{2} \\
& =\frac{\beta^{2}}{\varepsilon_{1}^{2 \beta} \varepsilon_{2}^{\beta}} \int_{0}^{t} \int_{0}^{t}\left\{\varepsilon_{2}^{\beta} \Phi_{s, r}\left(1, \varepsilon_{1}\right)-\varepsilon_{1}^{\beta} \Phi_{s, r}\left(2, \varepsilon_{1}, \varepsilon_{2}\right)\right\}(s r)^{\beta-1} d r d s \\
& \quad+\frac{\beta^{2}}{\varepsilon_{1}^{\beta} \varepsilon_{2}^{2 \beta}} \int_{0}^{t} \int_{0}^{t}\left\{\varepsilon_{1}^{\beta} \Phi_{s, r}\left(1, \varepsilon_{2}\right)-\varepsilon_{2}^{\beta} \Phi_{s, r}\left(2, \varepsilon_{1}, \varepsilon_{2}\right)\right\}(s r)^{\beta-1} d r d s
\end{aligned}
$$

for all $t \geq 0$ and $\varepsilon_{1}, \varepsilon_{2}>0$. Thus, in order to see that $\left\{I_{\varepsilon}^{-}(f, t), \varepsilon>0\right\}$ is a Cauchy sequence in $L^{2}(\Omega)$, we show that

$$
\frac{1}{\varepsilon_{i}^{2 \beta} \varepsilon_{j}^{\beta}} \int_{0}^{t} \int_{0}^{t}\left\{\varepsilon_{j}^{\beta} \Phi_{s, r}\left(1, \varepsilon_{i}\right)-\varepsilon_{i}^{\beta} \Phi_{s, r}\left(2, \varepsilon_{1}, \varepsilon_{2}\right)\right\}(s r)^{\beta-1} d r d s \longrightarrow 0
$$


for all $i, j \in\{1,2\}, i \neq j$, as $\varepsilon_{1}, \varepsilon_{2} \rightarrow 0$. Without loss of generality, we assume that $\varepsilon_{1}>\varepsilon_{2}$. By the proof of (i), it follows that

$$
\begin{aligned}
\Phi_{s, r}(1, \varepsilon)= & E\left[W_{s}^{\alpha}\left(W_{r+\varepsilon}^{\alpha}-W_{r}^{\alpha}\right)\right] E\left[W_{s}^{\alpha}\left(W_{s+\varepsilon}^{\alpha}-W_{s}^{\alpha}\right)\right] E\left[f^{\prime \prime}\left(W_{s}^{\alpha}\right) f\left(W_{r}^{\alpha}\right)\right] \\
& +E\left[W_{s}^{\alpha}\left(W_{r+\varepsilon}^{\alpha}-W_{r}^{\alpha}\right)\right] E\left[W_{r}^{\alpha}\left(W_{s+\varepsilon}^{\alpha}-W_{s}^{\alpha}\right)\right] E\left[f^{\prime}\left(W_{s}^{\alpha}\right) f^{\prime}\left(W_{r}^{\alpha}\right)\right] \\
& +E\left[W_{r}^{\alpha}\left(W_{r+\varepsilon}^{\alpha}-W_{r}^{\alpha}\right)\right] E\left[W_{s}^{\alpha}\left(W_{s+\varepsilon}^{\alpha}-W_{s}^{\alpha}\right)\right] E\left[f^{\prime}\left(W_{s}^{\alpha}\right) f^{\prime}\left(W_{r}^{\alpha}\right)\right] \\
& +E\left[W_{r}^{\alpha}\left(W_{r+\varepsilon}^{\alpha}-W_{r}^{\alpha}\right)\right] E\left[W_{r}^{\alpha}\left(W_{s+\varepsilon}^{\alpha}-W_{s}^{\alpha}\right)\right] E\left[f\left(W_{s}^{\alpha}\right) f^{\prime \prime}\left(W_{r}^{\alpha}\right)\right] \\
& +E\left[\left(W_{r+\varepsilon}^{\alpha}-W_{r}^{\alpha}\right)\left(W_{s+\varepsilon}^{\alpha}-W_{s}^{\alpha}\right)\right] E\left[f\left(W_{s}^{\alpha}\right) f\left(W_{r}^{\alpha}\right)\right]
\end{aligned}
$$

and

$$
\begin{aligned}
\Phi_{s, r}\left(2, \varepsilon_{1}, \varepsilon_{2}\right)= & E\left[W_{s}^{\alpha}\left(W_{r+\varepsilon_{2}}^{\alpha}-W_{r}^{\alpha}\right)\right] E\left[W_{s}^{\alpha}\left(W_{s+\varepsilon_{1}}^{\alpha}-W_{s}^{\alpha}\right)\right] E\left[f^{\prime \prime}\left(W_{s}^{\alpha}\right) f\left(W_{r}^{\alpha}\right)\right] \\
& +E\left[W_{s}^{\alpha}\left(W_{r+\varepsilon_{2}}^{\alpha}-W_{r}^{\alpha}\right)\right] E\left[W_{r}^{\alpha}\left(W_{s+\varepsilon_{1}}^{\alpha}-W_{s}^{\alpha}\right)\right] E\left[f^{\prime}\left(W_{s}^{\alpha}\right) f^{\prime}\left(W_{r}^{\alpha}\right)\right] \\
& +E\left[B_{r}\left(W_{r+\varepsilon_{2}}^{\alpha}-B_{r}\right)\right] E\left[W_{s}^{\alpha}\left(W_{s+\varepsilon_{1}}^{\alpha}-B_{s}\right)\right] E\left[f^{\prime}\left(W_{s}^{\alpha}\right) f^{\prime}\left(W_{r}^{\alpha}\right)\right] \\
& +E\left[W_{r}^{\alpha}\left(W_{r+\varepsilon_{2}}^{\alpha}-W_{r}^{\alpha}\right)\right] E\left[W_{r}^{\alpha}\left(W_{s+\varepsilon_{1}}^{\alpha}-W_{s}^{\alpha}\right)\right] E\left[f\left(W_{s}^{\alpha}\right) f^{\prime \prime}\left(W_{r}^{\alpha}\right)\right] \\
& +E\left[\left(W_{s+\varepsilon_{1}}^{\alpha}-W_{s}^{\alpha}\right)\left(W_{r+\varepsilon_{2}}^{\alpha}-W_{r}^{\alpha}\right)\right] E\left[f\left(W_{s}^{\alpha}\right) f\left(W_{r}^{\alpha}\right)\right] .
\end{aligned}
$$

For $\varepsilon_{1}, \varepsilon_{2}, \varepsilon, s, r>0$ and $j \in\{1,2\}$. Denote

$$
\begin{aligned}
A_{1}(s, r, \varepsilon, j):= & \varepsilon_{j}^{\beta} E\left[\left(W_{r+\varepsilon}^{\alpha}-W_{r}^{\alpha}\right)\left(W_{s+\varepsilon}^{\alpha}-W_{s}^{\alpha}\right)\right] \\
& -\varepsilon^{\beta} E\left[\left(W_{s+\varepsilon_{1}}^{\alpha}-W_{s}^{\alpha}\right)\left(W_{r+\varepsilon_{2}}^{\alpha}-W_{r}^{\alpha}\right)\right], \\
A_{2}(s, r, \varepsilon, j):= & \varepsilon_{j}^{\beta} E\left[W_{r}^{\alpha}\left(W_{r+\varepsilon}^{\alpha}-W_{r}^{\alpha}\right)\right] E\left[W_{r}^{\alpha}\left(W_{s+\varepsilon}^{\alpha}-W_{s}^{\alpha}\right)\right] \\
& -\varepsilon^{\beta} E\left[W_{r}^{\alpha}\left(W_{r+\varepsilon_{2}}^{\alpha}-W_{r}^{\alpha}\right)\right] E\left[W_{r}^{\alpha}\left(W_{s+\varepsilon_{1}}^{\alpha}-W_{s}^{\alpha}\right)\right], \\
A_{3}(s, r, \varepsilon, j):= & \varepsilon_{j}^{\beta} E\left[W_{s}^{\alpha}\left(W_{r+\varepsilon}^{\alpha}-W_{r}^{\alpha}\right)\right] E\left[W_{s}^{\alpha}\left(W_{s+\varepsilon}^{\alpha}-W_{s}^{\alpha}\right)\right] \\
& -\varepsilon^{\beta} E\left[W_{s}^{\alpha}\left(W_{r+\varepsilon_{2}}^{\alpha}-W_{r}^{\alpha}\right)\right] E\left[W_{s}^{\alpha}\left(W_{s+\varepsilon_{1}}^{\alpha}-W_{s}^{\alpha}\right)\right], \\
A_{41}(s, r, \varepsilon, j):= & \varepsilon_{j}^{\beta} E\left[W_{r}^{\alpha}\left(W_{r+\varepsilon}^{\alpha}-W_{r}^{\alpha}\right)\right] E\left[W_{s}^{\alpha}\left(W_{s+\varepsilon}^{\alpha}-W_{s}^{\alpha}\right)\right] \\
& -\varepsilon^{\beta} E\left[W_{r}^{\alpha}\left(W_{r+\varepsilon_{2}}^{\alpha}-W_{r}^{\alpha}\right)\right] E\left[W_{s}^{\alpha}\left(W_{s+\varepsilon_{1}}^{\alpha}-W_{s}^{\alpha}\right)\right], \\
A_{42}(s, r, \varepsilon, j):= & \varepsilon_{j}^{\beta} E\left[W_{s}^{\alpha}\left(W_{r+\varepsilon}^{\alpha}-W_{r}^{\alpha}\right)\right] E\left[W_{r}^{\alpha}\left(W_{s+\varepsilon}^{\alpha}-W_{s}^{\alpha}\right)\right] \\
& -\varepsilon^{\beta} E\left[W_{s}^{\alpha}\left(W_{r+\varepsilon_{2}}^{\alpha}-W_{r}^{\alpha}\right)\right] E\left[W_{r}^{\alpha}\left(W_{s+\varepsilon_{1}}^{\alpha}-W_{s}^{\alpha}\right)\right] .
\end{aligned}
$$

One obtains

$$
\begin{aligned}
\varepsilon_{j}^{\beta} \Phi_{s, r}\left(1, \varepsilon_{i}\right)-\varepsilon_{i}^{\beta} \Phi_{s, r}\left(2, \varepsilon_{1}, \varepsilon_{2}\right) \\
=E\left[f\left(W_{s}^{\alpha}\right) f\left(W_{r}^{\alpha}\right)\right] A_{1}\left(s, r, \varepsilon_{i}, j\right) \\
+E\left[f\left(W_{s}^{\alpha}\right) f^{\prime \prime}\left(W_{r}^{\alpha}\right)\right] A_{2}\left(s, r, \varepsilon_{i}, j\right)+E\left[f^{\prime \prime}\left(W_{s}^{\alpha}\right) f\left(W_{r}^{\alpha}\right)\right] A_{3}\left(s, r, \varepsilon_{i}, j\right) \\
+E\left[f^{\prime}\left(W_{s}^{\alpha}\right) f^{\prime}\left(W_{r}^{\alpha}\right)\right]\left(A_{41}\left(s, r, \varepsilon_{i}, j\right)+A_{42}\left(s, r, \varepsilon_{i}, j\right)\right)
\end{aligned}
$$


with $i \neq j$ and $i, j \in\{1,2\}$. In the sequel, we prove the convergence of (4.7). By symmetry, one only needs to show that, for $i=1, j=2$, the convergence holds. We divide the proof into four steps.

Step I. The following convergence holds:

$$
\lim _{\varepsilon_{1}, \varepsilon_{2} \rightarrow 0} \frac{1}{\varepsilon_{1}^{2 \beta} \varepsilon_{2}^{\beta}} \int_{0}^{t} \int_{0}^{t} A_{1}\left(s, r, \varepsilon_{1}, 2\right) E\left[f\left(W_{s}^{\alpha}\right) f\left(W_{r}^{\alpha}\right)\right](s r)^{\beta-1} d r d s=0 .
$$

It is clear that, for $i, j \in\{1,2\}, 0<|s-r|<\varepsilon_{i} \wedge \varepsilon_{j} \leq 1,0<\lambda<1-\beta$, we have

$$
\begin{aligned}
& \left|E\left[\left(W_{s+\varepsilon_{i}}^{\alpha}-W_{s}^{\alpha}\right)\left(W_{r+\varepsilon_{j}}^{\alpha}-W_{r}^{\alpha}\right)\right]\right| \\
& \leq \sqrt{E\left[\left(W_{s+\varepsilon_{i}}^{\alpha}-W_{s}^{\alpha}\right)^{2} E\left(W_{r+\varepsilon_{j}}^{\alpha}-W_{r}^{\alpha}\right)^{2}\right]} \\
& \quad \leq C \varepsilon_{i}^{\beta / 2} \varepsilon_{j}^{\beta / 2} \leq C \frac{\varepsilon_{i}^{\beta+\lambda} \varepsilon_{j}^{\beta}}{|s-r|^{\beta+\lambda}} .
\end{aligned}
$$

Combining this inequality with (2.7) (by taking $\gamma=\frac{\beta+\lambda}{2-\beta}$ ), we have

$$
\begin{aligned}
& \left|E\left[\left(W_{s+\varepsilon_{1}}^{\alpha}-W_{s}^{\alpha}\right)\left(W_{r+\varepsilon_{1}}^{\alpha}-W_{r}^{\alpha}\right)\right]\right| \\
& \quad \leq C\left(\frac{\varepsilon_{1}^{2 \beta+\lambda}}{|s-r|^{\beta+\lambda}} 1_{\left\{|s-r|>\varepsilon_{1}\right\}}+\frac{\varepsilon_{1}^{2 \beta+\lambda}}{|s-r|^{\beta+\lambda}} 1_{\left\{0<|s-r| \leq \varepsilon_{1}\right\}}\right) \leq C \frac{\varepsilon_{1}^{2 \beta+\lambda}}{|s-r|^{\beta+\lambda}}
\end{aligned}
$$

and

$$
\begin{aligned}
& \left|E\left[\left(W_{s+\varepsilon_{1}}^{\alpha}-W_{s}^{\alpha}\right)\left(W_{r+\varepsilon_{2}}^{\alpha}-W_{r}^{\alpha}\right)\right]\right| \\
& \quad \leq C\left(\frac{\varepsilon_{1}^{\beta+\frac{\gamma}{2}} \varepsilon_{2}^{\beta+\frac{\lambda}{2}}}{|s-r|^{\beta+\lambda}} 1_{\left\{|s-r|>\varepsilon_{2}\right\}}+\frac{\varepsilon_{1}^{\beta+\lambda} \varepsilon_{2}^{\beta}}{|s-r|^{\beta+\lambda}} 1_{\left\{0<|s-r| \leq \varepsilon_{2}\right\}}\right) \leq C \frac{\varepsilon_{1}^{\beta+\lambda} \varepsilon_{2}^{\beta}}{|s-r|^{\beta+\lambda}}
\end{aligned}
$$

for $0<\lambda<1-\beta$ and $|s-r|>0$. We deduce that

$$
\frac{1}{\varepsilon_{1}^{2 \beta} \varepsilon_{2}^{\beta}}\left|A_{1}\left(s, r, \varepsilon_{1}, 2\right)\right| \leq C_{H} \frac{\varepsilon_{1}^{\lambda}}{|s-r|^{\beta+\lambda}} \longrightarrow 0 \quad\left(\varepsilon_{1}, \varepsilon_{2} \rightarrow 0\right)
$$

for $0<\lambda<1-\beta$ and $s, r>0$.

Besides, from the above proof, we also have

$$
\begin{aligned}
\frac{1}{\varepsilon_{1}^{2 \beta} \varepsilon_{2}^{\beta}}\left|A_{1}\left(s, r, \varepsilon_{1}, 2\right)\right| \leq & \frac{1}{\varepsilon_{1}^{2 \beta}}\left|E\left[\left(W_{s+\varepsilon_{1}}^{\alpha}-W_{s}^{\alpha}\right)\left(W_{r+\varepsilon_{1}}^{\alpha}-W_{r}^{\alpha}\right)\right]\right| \\
& +\frac{1}{\varepsilon_{1}^{\beta} \varepsilon_{2}^{\beta}}\left|E\left[\left(W_{s+\varepsilon_{1}}^{\alpha}-W_{s}^{\alpha}\right)\left(W_{r+\varepsilon_{2}}^{\alpha}-W_{r}^{\alpha}\right)\right]\right| \\
\leq & C \frac{1}{|s-r|^{\beta}}
\end{aligned}
$$

for all $\varepsilon_{1}, \varepsilon_{2}>0,|s-r|>0$, and

$$
\int_{0}^{t} \int_{0}^{t} \frac{1}{|s-r|^{\beta}}\left|E\left[f\left(W_{s}^{\alpha}\right) f\left(W_{r}^{\alpha}\right)\right]\right|(s r)^{\beta_{2}-1} d r d s \leq C\|f\|_{\mathscr{H}}^{2}
$$


for every $0<\varepsilon_{1}, \varepsilon_{2}<1$. By Lebesgue's dominated convergence theorem it follows that (4.8) is convergent.

Step II. We show that the following convergence holds:

$$
\lim _{\varepsilon_{1}, \varepsilon_{2} \rightarrow 0} \frac{1}{\varepsilon_{1}^{2 \beta} \varepsilon_{2}^{\beta}} \int_{0}^{t} \int_{0}^{t} A_{2}\left(s, r, \varepsilon_{1}, 2\right) E\left[f\left(W_{s}^{\alpha}\right) f^{\prime \prime}\left(W_{r}^{\alpha}\right)\right](s r)^{\beta-1} d r d s=0
$$

From Lemma 4.1 and Lemma 2.2, we obtain, for $\varepsilon_{1}, \varepsilon_{2}>0$,

$$
\frac{1}{\varepsilon_{1}^{2 \beta} \varepsilon_{2}^{\beta}}\left|A_{2}\left(s, r, \varepsilon_{1}, 2\right)\right| \leq C
$$

and

$$
\int_{0}^{t} \int_{0}^{t}\left|E\left[f\left(W_{s}^{\alpha}\right) f^{\prime \prime}\left(W_{r}^{\alpha}\right)\right]\right|(s r)^{\beta-1} d r d s \leq C\|f\|_{\mathscr{H}}^{2}
$$

On the other hand, by the fact that

$$
b^{\alpha}-a^{\alpha} \leq b^{\alpha-\gamma}(b-a)^{\gamma}
$$

with $0<\alpha \leq \gamma \leq 1, b>a>0$, and Lemma 2.2, we get

$$
\begin{aligned}
\frac{1}{\varepsilon_{1}^{2 \beta} \varepsilon_{2}^{\beta}}\left|A_{2}\left(s, r, \varepsilon_{1}, 2\right)\right|= & \frac{1}{\varepsilon_{1}^{2 \beta} \varepsilon_{2}^{\beta}}\left|E\left[W_{r}^{\alpha}\left(W_{s+\varepsilon_{1}}^{\alpha}-W_{s}^{\alpha}\right)\right]\right| \\
& \cdot\left|\varepsilon_{2}^{\beta} E\left[W_{r}^{\alpha}\left(W_{r+\varepsilon_{1}}^{\alpha}-W_{r}^{\alpha}\right)\right]-\varepsilon_{1}^{\beta} E\left[W_{r}^{\alpha}\left(W_{r+\varepsilon_{2}}^{\alpha}-W_{r}^{\alpha}\right)\right]\right| \\
= & \frac{1}{\varepsilon_{1}^{2 \beta} \varepsilon_{2}^{\beta}}\left|E\left[W_{r}^{\alpha}\left(W_{s+\varepsilon_{1}}^{\alpha}-W_{s}^{\alpha}\right)\right]\right| \\
& \cdot \frac{1}{2}\left|\varepsilon_{2}^{\beta}\left(\left(r+\varepsilon_{1}\right)^{\beta}-r^{\beta}\right)-\varepsilon_{1}^{\beta}\left(\left(r+\varepsilon_{2}\right)^{\beta}-r^{\beta}\right)\right| \\
\leq & r^{\beta-\gamma} \varepsilon_{1}^{\gamma-\beta} \longrightarrow 0 \quad\left(\varepsilon_{1}, \varepsilon_{2} \rightarrow 0\right)
\end{aligned}
$$

for all $r>0$ and $\beta<\gamma \leq 1$, by the Lebesgue dominated convergence theorem it follows that the convergence of (4.9) hold.

Step III. We show that the following convergence holds:

$$
\lim _{\varepsilon_{1}, \varepsilon_{2} \rightarrow 0} \frac{1}{\varepsilon_{1}^{2 \beta} \varepsilon_{2}^{\beta}} \int_{0}^{t} \int_{0}^{t} A_{3}\left(s, r, \varepsilon_{1}, 2\right) E\left[f^{\prime \prime}\left(W_{s}^{\alpha}\right) f\left(W_{r}^{\alpha}\right)\right](s r)^{\beta-1} d r d s=0
$$

By (4.10) we get

$$
\begin{aligned}
\left|E\left[W_{s}^{\alpha}\left(W_{r+\varepsilon}^{\alpha}-W_{r}^{\alpha}\right)\right]\right| & =\frac{1}{2}\left|(r+\varepsilon)^{\beta}-\right| s-r-\left.\varepsilon\right|^{\beta}-r^{\beta}+|s-r|^{\beta} \mid \\
& \leq \frac{1}{2}\left|(r+\varepsilon)^{\beta}-r^{\beta}\right|+\frac{1}{2}|| s-\left.r\right|^{\beta}+|s-r-\varepsilon|^{\beta} \mid \\
& \leq \frac{1}{2}\left(r^{\beta-\gamma}+|s-r|^{\beta-\gamma}\right) \varepsilon^{\beta}
\end{aligned}
$$


for $\beta \leq \gamma \leq 1, \varepsilon>0$, and $|s-r|>0$. By Lemma 2.2 and (4.11) it follows that, for all $s, r>0$, $\beta<\gamma \leq 1$,

$$
\begin{aligned}
\frac{1}{\varepsilon_{1}^{2 \beta} \varepsilon_{2}^{\beta}}\left|A_{3}\left(s, r, \varepsilon_{1}, 2\right)\right|= & \frac{1}{\varepsilon_{1}^{2 \beta} \varepsilon_{2}^{\beta}}\left|E\left[W_{s}^{\alpha}\left(W_{s+\varepsilon_{1}}^{\alpha}-W_{s}^{\alpha}\right)\right]\right| \\
& \cdot\left|\varepsilon_{2}^{\beta} E\left[W_{s}^{\alpha}\left(W_{r+\varepsilon_{1}}^{\alpha}-W_{r}^{\alpha}\right)\right]-\varepsilon_{1}^{\beta} E\left[W_{s}^{\alpha}\left(W_{r+\varepsilon_{2}}^{\alpha}-W_{r}^{\alpha}\right)\right]\right| \\
\leq & r^{\beta-\gamma} \varepsilon^{\gamma-\beta} 1_{\{s=r\}}+\left(r^{\beta-\gamma}+|s-r|^{\beta-\gamma}\right) \varepsilon^{\gamma-\beta} 1_{\{|s-r|>0\}} \longrightarrow 0
\end{aligned}
$$

as $\varepsilon_{1}, \varepsilon_{2} \rightarrow 0$. Noting that for all $\varepsilon_{1}, \varepsilon_{2}>0$,

$$
\int_{0}^{t} \int_{0}^{t}\left|A_{3}\left(s, r, \varepsilon_{1}, 2\right) E\left[f^{\prime \prime}\left(W_{s}^{\alpha}\right) f\left(W_{r}^{\alpha}\right)\right]\right|(s r)^{\beta-1} d r d s \leq C_{H}\|f\|_{\mathscr{H}}^{2}
$$

we obtain the convergence (4.12) by using Lebesgue's dominated convergence theorem.

Step IV. We show that the following convergence holds:

$$
\begin{aligned}
& \lim _{\lim _{\varepsilon_{1}, \varepsilon_{2} \rightarrow 0}} \frac{1}{\varepsilon_{1}^{2 \beta} \varepsilon_{2}^{\beta}} \int_{0}^{t} \int_{0}^{t}\left(A_{41}\left(s, r, \varepsilon_{1}, 2\right)+A_{42}\left(s, r, \varepsilon_{1}, 2\right)\right) \\
& \cdot E\left[f^{\prime}\left(W_{s}^{\alpha}\right) f^{\prime}\left(W_{r}^{\alpha}\right)\right](s r)^{\beta-1} d r d s=0 .
\end{aligned}
$$

For $r>0$ and $\beta<\gamma \leq 1$, from Step II it follows that

$$
\begin{aligned}
\frac{1}{\varepsilon_{1}^{2 \beta} \varepsilon_{2}^{\beta}}\left|A_{41}\left(s, r, \varepsilon_{1}, 2\right)\right| \leq & \frac{1}{\varepsilon_{1}^{2 \beta} \varepsilon_{2}^{\beta}}\left|E\left[W_{s}^{\alpha}\left(W_{s+\varepsilon_{1}}^{\alpha}-W_{s}^{\alpha}\right)\right]\right| \\
& \cdot\left|\varepsilon_{2}^{\beta} E\left[W_{r}^{\alpha}\left(W_{r+\varepsilon_{1}}^{\alpha}-W_{r}^{\alpha}\right)\right]-\varepsilon_{1}^{\beta} E\left[W_{r}^{\alpha}\left(W_{r+\varepsilon_{2}}^{\alpha}-W_{r}^{\alpha}\right)\right]\right| \\
\leq & r^{\beta-\gamma} \varepsilon_{1}^{\gamma-\beta} \longrightarrow 0
\end{aligned}
$$

as $\varepsilon_{1}, \varepsilon_{2} \rightarrow 0$. for $s, r>0$ and $\beta<\gamma \leq 1$, from Step III we get

$$
\begin{aligned}
\frac{1}{\varepsilon_{1}^{2 \beta} \varepsilon_{2}^{\beta}}\left|A_{42}\left(s, r, \varepsilon_{1}, 2\right)\right|= & \frac{1}{\varepsilon_{1}^{\beta \beta} \varepsilon_{2}^{\beta}}\left|E\left[W_{r}^{\alpha}\left(W_{s+\varepsilon_{1}}^{\alpha}-W_{s}^{\alpha}\right)\right]\right| \\
& \cdot\left|\varepsilon_{2}^{\beta} E\left[W_{s}^{\alpha}\left(W_{r+\varepsilon_{1}}^{\alpha}-W_{r}^{\alpha}\right)\right]-\varepsilon_{1}^{\beta} E\left[W_{s}^{\alpha}\left(W_{r+\varepsilon_{2}}^{\alpha}-W_{r}^{\alpha}\right)\right]\right| \\
\leq & r^{\beta-\gamma} \varepsilon^{\gamma-\beta} 1_{\{s=r\}}+\left(r^{\beta-\gamma}+|s-r|^{\beta-\gamma}\right) \varepsilon^{\gamma-\beta} 1_{\{|s-r|>0\}} \longrightarrow 0
\end{aligned}
$$

as $\varepsilon_{1}, \varepsilon_{2} \rightarrow 0$. On the other hand, for all $\varepsilon_{1}, \varepsilon_{2}>0$, we have

$$
\begin{aligned}
& \frac{1}{\varepsilon_{1}^{2 \beta} \varepsilon_{2}^{\beta}} \int_{0}^{t} \int_{0}^{t}\left|A_{41}\left(s, r, \varepsilon_{1}, 2\right)+A_{42}\left(s, r, \varepsilon_{1}, 2\right)\right|\left|E\left[f^{\prime}\left(W_{s}^{\alpha}\right) f^{\prime}\left(W_{r}^{\alpha}\right)\right]\right|(s r)^{\beta-1} d r d s \\
& \quad \leq C \int_{0}^{t} \int_{0}^{t}\left|E\left[f^{\prime}\left(W_{s}^{\alpha}\right) f^{\prime}\left(W_{r}^{\alpha}\right)\right]\right|(s r)^{\beta-1} d r d s \leq C\|f\|_{\mathscr{H}}^{2}
\end{aligned}
$$

by Lebesgue's dominated convergence theorem, the convergence of (4.13) follows.

Therefore, $\left\{I_{\varepsilon}^{-}(f, t), \varepsilon>0\right\}$ is a Cauchy sequence in $L^{2}(\Omega)$. Similarly, we can also show that $\left\{I_{\varepsilon}^{+}(f, t), \varepsilon>0\right\}$ is a Cauchy sequence in $L^{2}(\Omega)$, and the lemma follows. 
Theorem 4.1 Let $g \in \mathscr{H}$. Then the generalized quadratic covariation of $W^{\alpha}$ and $g\left(W^{\alpha}\right)$ exists in $L^{2}(\Omega)$ and for all $t \in[0,1]$,

$$
E|| g\left(W^{\alpha}\right),\left.\left.W^{\alpha}\right|_{t} ^{(\alpha)}\right|^{2} \leq C\|g\|_{\mathscr{H}}^{2} .
$$

Proof Let $g \in \mathscr{H}$ be given. Since $\mathscr{E}$ is dense in $\mathscr{H}$, we can take the sequence $\left\{g_{\Delta, n}\right\} \subset \mathscr{E}$ such that $g_{\Delta, n} \rightarrow f$ in $\mathscr{H}$. If the theorem is true for all functions belonging to $\mathscr{E}$, then for all $\varepsilon_{1}, \varepsilon_{2}>0$ and $n \geq 1$, we get

$$
E\left|I_{\varepsilon_{1}}(g, t)-I_{\varepsilon_{2}}(g, t)\right|^{2} \leq C\left\|g-g_{\triangle, n}\right\|_{\mathscr{H}}^{2}+C E\left|I_{\varepsilon_{1}}\left(g_{\triangle, n}, t\right)-I_{\varepsilon_{2}}\left(g_{\triangle, n}, t\right)\right|^{2} .
$$

Thus, to end the proof, we only need to verify the theorem for $g \in \mathscr{E}$.

Let $g_{\Delta}(y)=\sum_{i} b_{i} 1_{\left(y_{i-1}, y_{i}\right]}(y)$. It is obvious that $g_{\triangle}$ is bounded and left continuous. Consider the function $\xi$ defined on $\mathbb{R}$ by

$$
\xi(x):= \begin{cases}k e^{\frac{1}{(y-1)^{2}-1}}, & y \in(0,2), \\ 0, & \text { otherwise }\end{cases}
$$

where $k$ is a normalizing constant satisfying $\int_{\mathbb{R}} \xi(y) d y=1$. For all $y \in \mathbb{R}$, we define the mollifiers and the corresponding sequence of smooth functions, respectively, by

$$
\begin{aligned}
& \xi_{n}(x):=n \xi(n x), \quad n=1,2, \ldots \\
& g_{n, \Delta}(y)=\int_{\mathbb{R}} g_{\Delta}(y-x) \xi_{n}(x) d x=\int_{0}^{2} g_{\Delta}\left(y-\frac{x}{n}\right) \xi(x) d x, \quad n=1,2, \ldots
\end{aligned}
$$

Then, for every $n, g_{n, \Delta} \in C_{0}^{\infty}(\mathbb{R}) \cap \mathscr{H}$ is bounded, and $g_{n, \Delta}$ converges to $g_{\triangle}$ in $\mathscr{H}$, as $n \rightarrow \infty$. Moreover, by the smooth approximation and the statement (i) we obtain

$$
E\left|I_{\varepsilon}^{ \pm}\left(g_{\Delta}, t\right)\right|^{2} \leq C\left\|g_{\Delta}\right\|_{\mathscr{H}}^{2}
$$

for all $t \in[0,1]$ and $\varepsilon>0$. Thus, for all $n$ and $\varepsilon_{1}, \varepsilon_{2}>0$, it follows that

$$
E\left|I_{\varepsilon_{1}}\left(g_{\Delta}, t\right)-I_{\varepsilon_{2}}\left(g_{\Delta}, t\right)\right|^{2} \leq C\left\|g_{\Delta}-g_{n, \Delta}\right\|_{\mathscr{H}}^{2}+C E\left|I_{\varepsilon_{1}}\left(g_{n, \Delta}, t\right)-I_{\varepsilon_{2}}\left(g_{n, \Delta}, t\right)\right|^{2},
$$

which implies that $\left\{I_{\varepsilon}\left(g_{\Delta}, t\right), \varepsilon>0\right\}$ is a Cauchy sequence by (ii). This means that Theorem 4.1 is true for $g \in \mathscr{E}$.

\section{Itô's formula and local time}

In this section, we investigate Itô's formula and the local time for the temporal process $W^{\alpha}$ by using the result of the previous sections. The first result is Itô's formula.

Theorem 5.1 Assume that $f \in \mathscr{H}$ is a left continuous function and $F$ is an absolutely continuous function satisfying $F^{\prime}=f$. then, for all $t \geq 0$, the Ito type formula

$$
F\left(W_{t}^{\alpha}\right)=G(0)+\int_{0}^{t} f\left(W_{s}^{\alpha}\right) d W_{s}^{\alpha}+\frac{1}{2^{1+\frac{1}{\alpha}}}\left\langle f\left(W^{\alpha}\right), W^{\alpha}\right\rangle_{t}^{(\alpha)}
$$

holds. 
This is an analog of Föllmer-Protter-Shiryayev's formula. For more details and works one may refer to Föllmer et al. [21], Eisenbaum [22], Russo-Vallois [18], Moret-Nualart [23], and the references therein. Recall that (see Alós et al. [16]) the Itô type formula

$$
F\left(W_{t}^{\alpha}\right)=F\left(W_{0}\right)+\int_{0}^{t} F^{\prime}\left(W_{s}^{\alpha}\right) d W_{s}^{\alpha}+\frac{1}{2} \kappa_{\alpha} \int_{0}^{t} F^{\prime \prime}\left(W_{s}^{\alpha}\right) d s^{\frac{\alpha-1}{\alpha}}
$$

holds for all $F \in C^{2}(\mathbb{R})$ satisfying the condition

$$
|F(y)|,\left|F^{\prime}(y)\right|,\left|F^{\prime \prime}(y)\right| \leq C e^{\beta y^{2}}, \quad y \in \mathbb{R}
$$

with $0 \leq \beta<\frac{1}{4 \kappa_{\alpha}} t^{\frac{1-\alpha}{\alpha}}$, where $\kappa_{\alpha}=\frac{\Gamma(1 / \alpha)}{2^{\frac{1}{\alpha}} \pi(\alpha-1)}$.

Proof of Theorem 5.1 If $f \in C^{1}(\mathbb{R})$, (5.1) is Itô's formula since

$$
\left\langle f\left(W^{\alpha}\right),\left.W^{\alpha}\right|_{t} ^{(\alpha)}=\frac{\Gamma(1 / \alpha)}{\pi(\alpha-1)} \int_{0}^{t} f^{\prime}\left(W_{s}^{\alpha}\right) d s^{\frac{\alpha-1}{\alpha}}\right.
$$

For $f \notin C^{1}(\mathbb{R})$, we can assume that $f$ is uniformly bounded by using a localization argument. In fact, for every $k \geq 0$, let

$$
\Omega_{k}=\left\{\sup _{0 \leq t \leq T}\left|W_{t}^{\alpha}\right|<k\right\}
$$

and

$$
f_{(k)}(x)= \begin{cases}f(x), & x \in[-k, k] \\ 0, & \text { otherwise }\end{cases}
$$

be a measurable function. It is clear that $f_{(k)} \in \mathscr{H}$ for every $k \geq 0$ and $f_{(k)}$ is uniformly bounded. Set $F_{(k)}=F$ on $[-k, k]$ and $\frac{d}{d x} F_{(k)}=f_{(k)}$. If the result of theorem is true for all uniformly bounded functions $f \in \mathscr{H}$, then the formula

$$
F_{(k)}\left(W_{t}^{\alpha}\right)=F_{(k)}(0)+\int_{0}^{t} f_{(k)}\left(W_{s}^{\alpha}\right) d W_{s}^{\alpha}+\frac{1}{2^{1+\frac{1}{\alpha}}}\left\langle f_{(k)}\left(W^{\alpha}\right),\left.W^{\alpha}\right|_{t} ^{(\alpha)}\right.
$$

holds on $\Omega_{k}$. Letting $k \rightarrow \infty$, we deduce the Itô formula (5.1).

Let $F^{\prime}=f \in \mathscr{H}$ be left continuous and uniformly bounded. For every $n \in \mathbb{N}^{+}$, we define

$$
F_{n}(y):=\int_{\mathbb{R}} F(y-x) \xi_{n}(x) d x, \quad y \in \mathbb{R}
$$

where $\xi_{n}, n=1,2, \ldots$, are defined by (4.16). It is obvious that $F_{n} \in C^{\infty}(\mathbb{R})$ for $n \geq 1$ and the Itô formula,

$$
F_{n}\left(W_{t}^{\alpha}\right)=F_{n}(0)+\int_{0}^{t} f_{n}\left(W_{s}^{\alpha}\right) d W_{s}^{\alpha}+\frac{1}{2} \kappa_{\alpha} \int_{0}^{t} f_{n}^{\prime}\left(W_{s}^{\alpha}\right) d s^{\frac{\alpha-1}{\alpha}}
$$


holds with $f_{n}=F_{n}^{\prime}$. By Lebesgue's dominated convergence theorem, we can show that, for each $x$,

$$
F_{n}(x) \longrightarrow F(x), \quad f_{n}(x) \longrightarrow f(x) \quad(n \rightarrow \infty),
$$

and $f_{n} \rightarrow f$ in $\mathscr{H}$. We further deduce that

$$
\kappa_{\alpha} \int_{0}^{t} f_{n}^{\prime}\left(W_{s}^{\alpha}\right) d s^{\frac{\alpha-1}{\alpha}}=2^{-\frac{1}{\alpha}}\left\langle f_{n}\left(W^{\alpha}\right),\left.W^{\alpha}\right|_{t} ^{(\alpha)} \longrightarrow 2^{-\frac{1}{\alpha}}\left\langle f\left(W^{\alpha}\right),\left.W^{\alpha}\right|_{t} ^{(\alpha)}\right.\right.
$$

and

$$
f_{n}\left(W_{t}^{\alpha}\right) \longrightarrow f\left(W_{t}^{\alpha}\right)
$$

in $L^{2}(\Omega)$ as $n \rightarrow \infty$. Therefore

$$
\begin{aligned}
\int_{0}^{t} f_{n}\left(W_{s}^{\alpha}\right) d W_{s}^{H} & =F_{n}\left(W_{t}^{H}\right)-F_{n}(0)-\frac{1}{2^{1+\frac{1}{\alpha}}}\left\langle f_{n}\left(W^{\alpha}\right), W^{\alpha}\right\rangle_{t}^{(\alpha)} \\
& \longrightarrow F\left(W_{t}^{\alpha}\right)-F(0)-\frac{1}{2^{1+\frac{1}{\alpha}}}\left\langle f\left(W^{\alpha}\right),\left.W^{\alpha}\right|_{t} ^{(\alpha)}\right.
\end{aligned}
$$

in $L^{2}(\Omega)$ as $n \rightarrow \infty$. The proof is completed.

At last, we investigate the local time of $W^{\alpha}$. It is well known that for any $x \in \mathbb{R}$ and any closed interval $I \subset \mathbb{R}_{+}$, the local time $L(x, I)$ of $W^{\alpha}$ is defined by

$$
\mu_{I}(A)=\int_{I} 1_{A}\left(W_{s}^{\alpha}\right) d s
$$

that is, the density of the occupation measure $\mu_{I}$. It is also shown (see Geman and Horowitz [24], Theorem 6.4) that the occupation density formula holds:

$$
\int_{I} g\left(W_{s}^{\alpha}, s\right) d s=\int_{\mathbb{R}} d x \int_{I} g(x, s) L(x, d s)
$$

where $g(x, t) \geq 0$ is a Borel function on $I \times \mathbb{R}$. Thus, by Theorem 21.9 in Geman-Horowitz [24] and Lemma 2.1, we get the following result.

Lemma 5.1 Let $L(x, t):=L(x,[0, t])$ be the local time of $W^{\alpha}$ at $x$. Then, for all $t \geq 0, L \in$ $L^{2}(\lambda \times P)$ and $(x, t) \mapsto L(x, t)$ is jointly continuous, where $\lambda$ denotes the Lebesgue measure. Moreover, the occupation formula

$$
\int_{0}^{t} \psi\left(W_{s}^{\alpha}, s\right) d s=\int_{\mathbb{R}} d x \int_{0}^{t} \psi(x, s) L(x, d s)
$$

holds for any $t \geq 0$ and every continuous and bounded function $\psi(x, t): \mathbb{R} \times \mathbb{R}_{+} \rightarrow \mathbb{R}$.

We now conclude this section with a comment on a generalized Bouleau-Yor identity. For more details and works one refers to Bouleau-Yor [25], Föllmer et al. [21], Eisenbaum 
[22], Feng-Zhao [26, 27], Rogers-Walsh [28], Peskir [29], Yan et al. [19, 30, 31], and the references therein.

For $t \geq 0$ and $x \in \mathbb{R}$, define the weighted local time $\mathscr{L}^{\alpha}$ by

$$
\begin{aligned}
\mathscr{L}^{\alpha}(x, t) & =\kappa_{\alpha} \int_{0}^{t} s^{\frac{\alpha-1}{\alpha}} d_{s} L(s, x) \\
& =\kappa_{\alpha} \int_{0}^{t} \delta\left(W_{s}^{\alpha}-x\right) d s^{\frac{\alpha-1}{\alpha}},
\end{aligned}
$$

where $\delta$ denotes the Dirac delta function.

In the sequel, we consider the integral

$$
\int_{\mathbb{R}} f(x) \mathscr{L}^{\alpha}(d x, t)
$$

and obtain the following Bouleau-Yor identity:

$$
\left\langle f\left(W^{\alpha}\right),\left.W^{\alpha}\right|_{t} ^{(\alpha)}=-2^{\frac{1}{\alpha}} \int_{\mathbb{R}} f(x) \mathscr{L}^{\alpha}(d x, t)\right.
$$

for all $f \in \mathscr{H}$.

Let $F(y)=(y-a)^{+}-(y-b)^{+}$, it is clear that $F$ is an absolutely continuous function with the derivative $F^{\prime}=1_{(a, b]} \in \mathscr{E}$, by using Itô's formula (5.1) it follows that, for all $t \geq 0$,

$$
\begin{aligned}
2^{-\frac{1}{\alpha}} 1_{(a, b]}\left(W^{\alpha}\right),\left.W^{\alpha}\right|_{t} ^{(\alpha)} & =2 F\left(W_{t}^{\alpha}\right)-2 F(0)-2 \int_{0}^{t} 1_{(a, b]}\left(W_{s}^{\alpha}\right) d W_{s}^{\alpha} \\
& =\mathscr{L}^{\alpha}(a, t)-\mathscr{L}^{\alpha}(b, t) .
\end{aligned}
$$

Therefore, by the linear property we obtain the following result.

Lemma 5.2 For any $f_{\triangle}=\sum_{j} f_{j} 1_{\left(a_{j-1}, a_{j}\right]} \in \mathscr{E}$, the integral

$$
\int_{\mathbb{R}} f_{\Delta}(x) \mathscr{L}^{\alpha}(d x, t):=\sum_{j} f_{j}\left[\mathscr{L}^{\alpha}\left(a_{j}, t\right)-\mathscr{L}^{\alpha}\left(a_{j-1}, t\right)\right]
$$

exists and

$$
2^{\frac{1}{\alpha}} \int_{\mathbb{R}} f_{\Delta}(x) \mathscr{L}^{\alpha}(d x, t)=-\left\langle f_{\Delta}\left(W^{\alpha}\right),\left.W^{\alpha}\right|_{t} ^{(\alpha)}\right.
$$

for all $t \geq 0$.

Noting that $\mathscr{E}$ is dense in $\mathscr{H}$, the definition of integration with respect to $x \mapsto \mathscr{L}^{\alpha}(x, t)$ can be extended to the elements of $\mathscr{H}$ in the following manner:

$$
\int_{\mathbb{R}} f(x) \mathscr{L}^{\alpha}(d x, t):=\lim _{n \rightarrow \infty} \int_{\mathbb{R}} f_{\triangle, n}(x) \mathscr{L}^{\alpha}(d x, t)
$$

in $L^{2}$ for $f \in \mathscr{H}$ provided $f_{\Delta, n} \rightarrow f$ in $\mathscr{H}$, as $n \rightarrow \infty$, where $\left\{f_{\triangle, n}\right\} \subset \mathscr{E}$. Thus, the integral

$$
\int_{\mathbb{R}} f(x) \mathscr{L}^{\alpha}(d x, t)
$$


is well defined and we obtain the desired Bouleau-Yor type identity

$$
\left\langle f\left(W^{\alpha}\right),\left.W^{\alpha}\right|_{t} ^{(\alpha)}=-2^{\frac{1}{\alpha}} \int_{\mathbb{R}} f(x) \mathscr{L}^{\alpha}(d x, t)\right.
$$

for all $f \in \mathscr{H}$.

Corollary 5.1 (Tanaka formula) For any $x \in \mathbb{R}$ we have

$$
\left|W_{t}^{\alpha}-x\right|=|x|+\int_{0}^{t} \operatorname{sign}\left(W_{s}^{\alpha}-x\right) d W_{s}^{\alpha}+\mathscr{L}^{\alpha}(x, t)
$$

\section{Competing interests}

The authors declare that they have no competing interests.

\section{Authors' contributions}

The main idea of this paper was proposed by LTY, YML and JC, LTY prepared the manuscript initially, JC performed all the steps of the proofs in this research. All authors read and approved the final manuscript.

\section{Author details}

'Department of Statistics, Anhui Normal University, 1 East Beijing Rd., Wuhu, 241000, P.R. China. ${ }^{2}$ Department of Mathematics, Donghua University, 2999 North Renmin Rd., Songjiang, Shanghai, 201620, P.R. China.

\section{Acknowledgements}

The authors are grateful to the anonymous referees and the associate editor for their valuable comments and suggestions to improve this manuscript. The project was sponsored by NSFC (Nos. 11571071, 11426036, 11401010), and Innovation Program of Shanghai Municipal Education Commission (No. 12ZZO63).

Received: 8 April 2016 Accepted: 23 June 2016 Published online: 01 July 2016

\section{References}

1. Swanson, J: Variations of the solution to a stochastic heat equation. Ann. Probab. 35, 2122-2159 (2007)

2. Pospisil, J, Tribe, R: Parameter estimation and exact variations for stochastic heat equations driven by space-time white noise. Stoch. Anal. Appl. 4, 830-856 (2007)

3. Sun, X, Yan, L, Yu, X: Quadratic covariations for the solution to a stochastic heat equation (2016, submitted). arXiv:1602.08796v2

4. Da Prato, G, Jentzen, A, Röckner, M: A mild Itô formula for SPDEs (2012). arXiv:1009.3526

5. Deya, A, Tindel, S: Malliavin calculus for fractional heat equation. In: Malliavin Calculus and Stochastic Analysis. Springer Proc. Math. Stat., pp. 361-384, vol. 34. Springer, New York (2013)

6. Denis, L: Solutions of stochastic partial differential equations considered as Dirichlet processes. Bernoulli 10, 783-827 (2004)

7. Gradinaru, M, Nourdin, I, Tindel, S: Ito's- and Tanaka's-type formulae for the stochastic heat equation: the linear case. J. Funct. Anal. 228, 114-143 (2005)

8. Ouahhabi, H, Tudor, CA: Additive functionals of the solution to fractional stochastic heat equation. J. Fourier Anal. Appl. 19, 777-791 (2013)

9. León, JA, Tindel, S: Itô formula for linear fractional PDEs. Stochastics 80, 427-450 (2008)

10. Torres, S, Tudor, CA, Viens, FG: Quadratic variations for the fractional-colored stochastic heat equation. Electron. J. Probab. 19, $76(2014)$

11. Tudor, CA, Xiao, Y: Sample paths of the solution to the fractional-colored stochastic heat equation. Stoch. Dyn. (2016). doi:10.1142/S0219493717500046

12. Tudor, CA: Chaos expansion and regularity of the local time of the solution to the stochastic heat equation with additive fractional-colored noise. Taiwan. J. Math. 17, 1765-1777 (2013)

13. Wu, D: On the solution process for a stochastic fractional partial differential equation driven by space-time white noise. Stat. Probab. Lett. 81, 1161-1172 (2011)

14. Zambotti, L: Itô-Tanaka's formula for stochastic partial differential equations driven by additive space-time white noise. In: Stochastic Partial Differential Equations and Applications - VII. Lect. Notes Pure Appl. Math., vol. 245, pp. 337-347 (2006)

15. Blumenthal, RM, Getoor, RK: Some theorems on stable processes. Trans. Am. Math. Soc. 95, 263-273 (1960)

16. Alós, E, Mazet, O, Nualart, D: Stochastic calculus with respect to Gaussian processes. Ann. Probab. 29, 766-801 (2001)

17. Nualart, D: Malliavin Calculus and Related Topics, 2nd edn. Springer, New York (2006)

18. Russo, F, Vallois, P: Itô formula for $\mathcal{C}^{1}$-functions of semimartingales. Probab. Theory Relat. Fields 104, 27-41 (1996)

19. Yan, L, Liu, J, Chen, C: The generalized quadratic covariation for fractional Brownian motion with Hurst index less than 1/2. Infin. Dimens. Anal. Quantum Probab. Relat. Top. 17(4), 1450030 (2011)

20. Gradinaru, M, Nourdin, I: Approximation at first and second order of $m$-order integrals of the fractional Brownian motion and of certain semimartingales. Electron. J. Probab. 8, 18 (2003)

21. Föllmer, H, Protter, PH, Shiryayev, AN: Quadratic covariation and an extension of Itô's formula. Bernoulli 1, 149-169 (1995) 
22. Eisenbaum, N: Integration with respect to local time. Potential Anal. 13, 303-328 (2000)

23. Moret, S, Nualart, D: Quadratic covariation and Itô's formula for smooth nondegenerate martingales. J. Theor. Probab. $13,193-224(2000)$

24. Geman, D, Horowitz, J: Occupation densities. Ann. Probab. 8, 1-67 (1980)

25. Bouleau, N, Yor, M: Sur la variation quadratique des temps locaux de certaines semimartingales. C. R. Acad. Sci. Paris, Sér. I Math. 292, 491-494 (1981)

26. Feng, CR, Zhao, HZ: Two-parameters p, q-variation paths and integrations of local times. Potential Anal. 25, 165-204 (2006)

27. Feng, C, Zhao, H: Local time rough path for Lévy processes. Electron. J. Probab. 15, 452-483 (2010)

28. Rogers, CG, Walsh, JB: Local time and stochastic area integrals. Ann. Probab. 19, 457-482 (1991)

29. Peskir, G: A change-of-variable formula with local time on curves. J. Theor. Probab. 18, 499-535 (2005)

30. Yan, L, Gao, B, Liu, J: The Bouleau-Yor identity for a bi-fBm. Stochastics 86, 382-414 (2014)

31. Yan, L, Liu, J, Yang, X: Integration with respect to fractional local time with Hurst index $1 / 2<H<1$. Potential Anal. 30, 115-138 (2009)

\section{Submit your manuscript to a SpringerOpen ${ }^{\circ}$} journal and benefit from:

- Convenient online submission

Rigorous peer review

- Immediate publication on acceptance

- Open access: articles freely available online

- High visibility within the field

- Retaining the copyright to your article 